KIAS-P01051

hep-ph/0111254

\title{
Model Independent Information On Solar Neutrino Oscillations
}

\author{
M.V. Garzelli \\ Dipartimento di Fisica Teorica, Università di Torino, \\ and INFN, Sezione di Torino, \\ Via P. Giuria 1, I-10125 Torino, Italy \\ C. Giuntif \\ INFN, Sezione di Torino, \\ and Dipartimento di Fisica Teorica, Università di Torino, \\ Via P. Giuria 1, I-10125 Torino, Italy \\ and \\ School of Physics, Korea Institute for Advanced Study, \\ Seoul 130-012, Korea
}

(Dated: 13 December 2001)

\begin{abstract}
We present the results of a Bayesian analysis of solar neutrino data in terms of $\nu_{e} \rightarrow \nu_{\mu, \tau}$ oscillations, independent from the Standard Solar Model predictions for the solar neutrino fluxes. We show that such a model independent analysis allows to constraint the values of the neutrino mixing parameters in limited regions around the usual SMA, LMA, LOW and VO regions. Furthermore, there is a strong indication in favor of large neutrino mixing and large values of $\Delta m^{2}$ (LMA region). We calculate also the allowed ranges of the neutrino fluxes and we show that they are in good agreement with the Standard Solar Model prediction. In particular, the ratio of the ${ }^{8} \mathrm{~B}$ flux with its Standard Solar Model prediction is constrained in the interval $[0.45,1.42]$ with $99.73 \%$ probability. Finally, we show that the hypothesis of no neutrino oscillations is strongly disfavored in a model independent way with respect to the hypothesis of neutrino oscillations.
\end{abstract}

PACS numbers: 26.65.+t, 14.60.Pq, 14.60.Lm

Keywords: Solar Neutrinos, Neutrino Physics, Statistical Methods

\section{INTRODUCTION}

An important model independent evidence in favor of transitions of solar electron neutrinos in other active states has been recently obtained from the comparison of the results of the Super-Kamiokande [1] and SNO [2] solar neutrino experiments [2, 3, 4, 5]. This evidence is supported [6] also by the results of the Homestake [7], GALLEX [8], SAGE [9] and GNO 10] experiments, that measured different suppressions of the solar electron neutrino flux with respect to the Standard Solar Model (SSM) 11 prediction.

The simplest and most natural mechanism that explains all solar neutrino data is $\nu_{e} \rightarrow \nu_{\mu, \tau}$ oscillations, due to neutrino masses and mixing (see [12, 13, 14, 15). Several groups have recently analyzed the data of solar neutrino experiments in terms of neutrino oscillations $13,16,17,18,19,20,21,22,22,24,25,26,27,28,29$. The authors of Refs. [3, 17, 18, 19, 20, 21, 24, 25, 29 found the allowed regions in the space of the neutrino mixing parameters assuming that the neutrino fluxes ${ }^{1}$ produced in the core of the sun are given by the BP2000 Standard Solar Model [11]. The authors of Refs. [17, 20, 24, 26, 29] performed analyses with the ${ }^{8} \mathrm{~B}$ neutrino flux considered as a free parameter to be determined from the data (in Ref. [20] also the ${ }^{7}$ Be neutrino flux has been considered as a free parameter, whereas the authors of Ref. [24] considered also the hep neutrino flux as a free parameter).

In this paper we calculate model independent allowed regions in the space of the neutrino mixing parameters. In the following, by "model independent" we mean independent from the solar model. In other words, we do not assume that the neutrino fluxes produced in the core of the sun are given by a Standard Solar Model.

\footnotetext{
*Electronic address: garzelli@to.infn.it

†Electronic address: giunti@to.infn.it; URL: http://www.to.infn.it/ giunti

1 The flux of solar neutrinos is composed by eight fluxes, pp, pep, hep, ${ }^{7} \mathrm{Be},{ }^{8} \mathrm{~B},{ }^{13} \mathrm{~N},{ }^{15} \mathrm{O},{ }^{17} \mathrm{~F}$, produced by the corresponding thermonuclear reactions in the core of the Sun (see [3]).
} 
We consider the simplest case of mixing of two neutrinos $\nu_{e}$ and $\nu_{a}$, where $\nu_{a}$ is a linear combination of $\nu_{\mu}$ and $\nu_{\tau}$ :

$$
\begin{aligned}
& \nu_{e}=\cos \vartheta \nu_{1}+\sin \vartheta \nu_{2}, \\
& \nu_{a}=-\sin \vartheta \nu_{1}+\cos \vartheta \nu_{2} .
\end{aligned}
$$

Here, $\vartheta$ is the mixing angle and $\nu_{1}, \nu_{2}$ are massive neutrinos with masses $m_{1}, m_{2}$, respectively. Neutrino oscillations depend on the mixing angle $\vartheta$ and the squared-mass difference $\Delta m^{2} \equiv m_{2}^{2}-m_{1}^{2}$ (see [12, 13, 14, 15]).

Let us notice, that the treatment of solar $\nu_{e} \rightarrow \nu_{a}$ transitions in the framework of the simple two-neutrino mixing scheme in Eq. (11) is valid with good approximation also in the case of mixing of three neutrinos $\left(\nu_{e}, \nu_{\mu}, \nu_{\tau}\right)$ with small $U_{e 3}$ [31, 32], as indicated by the results of the CHOOZ long-baseline $\bar{\nu}_{e}$ disappearance experiment [33]. Furthermore, the treatment is also valid in $3+1$ four-neutrino mixing schemes in which the sterile neutrino is practically decoupled from the active ones $\left(1-\left|U_{s 4}\right|^{2} \ll 1\right)[34]$ and $U_{e 3}$ is small, as indicated [35] by CHOOZ data.

Our statistical analysis of the data is Bayesian. We have already explained in Ref. [25] the advantages of using Bayesian Probability Theory in the analysis of solar neutrino data ${ }^{2}$ (for a general introduction to Bayesian Probability Theory see 37, 38, 39, 40, 41]). Another model independent analysis of solar neutrino data was performed several years ago $\sqrt{12}$ using a different statistical approach. Apart from the differences due to the statistical methods, the main difference with the analysis performed in Ref. [42] is the impressive increase of the quality and quantity of data, that, as we will show, allow now to obtain much more stringent model independent information.

As discussed in Ref. [25], the Bayesian analysis of solar neutrino data assuming the neutrino fluxes predicted by the Standard Solar Model takes into account the uncertainty of the fluxes in the covariance matrix of the least-squares function of the data, assuming a multi-normal distribution for the errors of the fluxes. This approach cannot be extended to the model independent analysis of solar neutrino data, in which the fluxes must be considered as free parameters that may be determined from the data in a similar way as the neutrino mixing parameters. Therefore, we have developed a new approach in which the neutrino fluxes are considered as unknown quantities with a flat prior probability distribution. The model independent information on neutrino mixing is calculated through marginalization of the posterior probability distribution with respect to the neutrino fluxes. We also derive information on each neutrino flux through marginalization of the posterior probability distribution with respect to the other neutrino fluxes and the oscillation parameters.

In the framework under consideration, the theoretical calculation of the rates measured in solar neutrino experiments depends on the neutrino mixing parameters $\tan ^{2} \vartheta$ and $\Delta m^{2}$, on the neutrino fluxes produced in the core of the sun by the thermonuclear reaction of the $p p$ and $\mathrm{CNO}$ cycles, and on the energy-averaged cross sections of neutrino interaction in the detectors. In order to have a uniform treatment of these uncertain theoretical quantities, we consider all of them as parameters that have some uncertainty, which is taken into account in the prior probability distribution. This approach is different from the standard one (see [25]), in which the uncertainties of the fluxes and cross sections are taken into account in the covariance matrix that determines the likelihood function (sampling distribution). However, we think that the new approach is equally, if not more, reasonable than the standard one, because the values of the fluxes and cross sections are part of our prior knowledge. In any case, the two approaches should give approximately the same result in the model dependent analysis in which the neutrino fluxes are assumed to be those predicted by the Standard Solar Model. We check this consistency in Section IV.

In the following it is convenient to group the solar neutrino fluxes in the array

$$
\Phi=\left(\Phi_{1}, \ldots, \Phi_{8}\right) .
$$

The neutrino flux $\Phi_{i}$ is produced in the $i^{\text {th }}$ thermonuclear reaction, with $i=1, \ldots, 8$ for the $p p, p e p, h e p,{ }^{7} \mathrm{Be},{ }^{8} \mathrm{~B}$, ${ }^{13} \mathrm{~N},{ }^{15} \mathrm{O},{ }^{17} \mathrm{~F}$ thermonuclear reactions, respectively.

In both the model dependent and independent analyses we perform a Rates Analysis that takes into account the total rates measured in the Homestake, GALLEX+GNO+SAGE, SNO, Super-Kamiokande experiments, and a Global Analysis in which the Super-Kamiokande total rate is replaced by the 38 bins of the Super-Kamiokande day and night electron energy spectra. Hence, calling $N_{\mathrm{S}}$ the number of solar data points, we have $N_{\mathrm{S}}=4$ in the Rates Analysis and $N_{\mathrm{S}}=41$ in the Global Analysis. We call $C_{i j}$ the energy-averaged cross section of detection of the $i^{\text {th }}$ neutrino flux for the $j^{\text {th }}$ solar data point, with $j=1, \ldots, N_{\mathrm{S}}$. We group these cross sections in the $8 \times N_{\mathrm{S}}$ matrix

$$
\mathbf{C}=\left(\begin{array}{ccc}
C_{11} & \cdots & C_{1 N_{\mathrm{S}}} \\
\vdots & \vdots & \vdots \\
C_{81} & \cdots & C_{8 N_{\mathrm{S}}}
\end{array}\right)
$$

2 Bayesian Probability Theory has been applied to the analysis of solar neutrino data also in Refs. 19, 36. 
Bayesian Probability Theory allows to calculate the posterior probability distribution $p\left(\tan ^{2} \vartheta, \Delta m^{2}, \Phi, \mathbf{C} \mid \mathrm{D}, \mathrm{I}\right)$ for the neutrino mixing parameters, the neutrino fluxes and the detection cross sections through Bayes Theorem:

$$
p\left(\tan ^{2} \vartheta, \Delta m^{2}, \Phi, \mathbf{C} \mid \mathrm{D}, \mathrm{I}\right)=\frac{p\left(\mathrm{D} \mid \tan ^{2} \vartheta, \Delta m^{2}, \Phi, \mathbf{C}, \mathrm{I}\right) p\left(\tan ^{2} \vartheta, \Delta m^{2} \mid \mathrm{I}\right) p(\Phi \mid \mathrm{I}) p(\mathbf{C} \mid \mathrm{I})}{p(\mathrm{D} \mid \mathrm{I})},
$$

where D represents the data and I represents all the background information and assumptions on solar physics, neutrino physics, etc. The sampling distribution $p\left(\mathrm{D} \mid \tan ^{2} \vartheta, \Delta m^{2}, \Phi, \mathbf{C}, \mathrm{I}\right)$ is also known as likelihood function. The prior distributions $p\left(\tan ^{2} \vartheta, \Delta m^{2} \mid \mathrm{I}\right), p(\Phi \mid \mathrm{I})$ and $p(\mathbf{C} \mid \mathrm{I})$ quantify our prior knowledge, or lack of knowledge, on the values of the mixing parameters, neutrino fluxes and detection cross sections, respectively. The probability distribution $p(\mathrm{D} \mid \mathrm{I})$ is known as global likelihood and acts as a normalization constant.

The prior probability distribution of mixing parameters, $p\left(\tan ^{2} \vartheta, \Delta m^{2} \mid \mathrm{I}\right)$, must be chosen in order to quantify appropriately the prior lack of knowledge on the values of the parameters $\tan ^{2} \vartheta$ and $\Delta m^{2}$. Assuming $\nu_{e} \rightarrow \nu_{\mu, \tau}$ oscillations (included in I), we know that solar neutrino data are sensitive to several different order of magnitude of $\tan ^{2} \vartheta$ and $\Delta m^{2}$, through vacuum oscillations 443] or resonant MSW transitions 44, 45. Therefore, the most reasonable non-informative prior probability distribution function, that we will use in the following, is a flat distribution in the $\log \left(\tan ^{2} \vartheta\right)-\log \left(\Delta m^{2}\right)$ plane. Indeed, the authors of Ref. [46] have chosen, independently, the same prior, which is consistent with the calculation of the credible regions for the neutrino oscillation parameters in $\operatorname{the} \log \left(\tan ^{2} \vartheta\right)-$ $\log \left(\Delta m^{2}\right)$ plane.

Using a flat prior probability distribution in the $\log \left(\tan ^{2} \vartheta\right)-\log \left(\Delta m^{2}\right)$ plane, Eq. (何) becomes

$$
p\left(\tan ^{2} \vartheta, \Delta m^{2}, \Phi, \mathbf{C} \mid \mathrm{D}, \mathrm{I}\right)=\frac{p\left(\mathrm{D} \mid \tan ^{2} \vartheta, \Delta m^{2}, \Phi, \mathbf{C}, \mathrm{I}\right) p(\Phi \mid \mathrm{I}) p(\mathbf{C} \mid \mathrm{I})}{\int d \log \left(\tan ^{2} \vartheta\right) \operatorname{d} \log \left(\Delta m^{2}\right) p\left(\mathrm{D} \mid \tan ^{2} \vartheta, \Delta m^{2}, \Phi, \mathbf{C}, \mathrm{I}\right) p(\Phi \mid \mathrm{I}) p(\mathbf{C} \mid \mathrm{I})},
$$

where we have expressed the global likelihood $p(\mathrm{D} \mid \mathrm{I})$ as the appropriate normalization constant. Integral probabilities must be calculated integrating $p\left(\tan ^{2} \vartheta, \Delta m^{2}, \Phi, \mathbf{C} \mid \mathrm{D}, \mathrm{I}\right)$ over $\operatorname{dlog}\left(\tan ^{2} \vartheta\right) \mathrm{d} \log \left(\Delta m^{2}\right) \mathrm{d} \Phi \mathrm{d} \mathbf{C}$.

The prior probability distribution of cross sections, $p(\mathbf{C} \mid \mathrm{I})$, represents our knowledge of the cross sections, which is reasonably accurate. Therefore, we take this prior probability distribution as a multinormal distribution as explained in Section III.

As explained in Subsection IVA, in the Standard Solar Model dependent analysis the prior probability distribution of neutrino fluxes, $p(\Phi \mid \mathrm{I})$, is a multinormal distribution. In the model independent analysis we consider a flat prior probability distribution of neutrino fluxes, as explained in Subsection $\mathrm{VA}$.

The posterior distribution for the oscillation parameters $\tan ^{2} \vartheta, \Delta m^{2}$ is obtained through marginalization of the posterior distribution (5) with respect to the neutrino fluxes and cross sections:

$$
p\left(\tan ^{2} \vartheta, \Delta m^{2} \mid \mathrm{D}, \mathrm{I}\right)=\int \mathrm{d} \Phi \int \mathrm{d} \mathbf{C} p\left(\tan ^{2} \vartheta, \Delta m^{2}, \Phi, \mathbf{C} \mid \mathrm{D}, \mathrm{I}\right) .
$$

Using this posterior distribution, we calculate Bayesian credible regions $^{3}$ in the plane of the oscillation parameters $\tan ^{2} \vartheta$ and $\Delta m^{2}$.

In both the Rates Analysis and the Global Analysis we consider also the data of the CHOOZ experiment, that are important because they exclude large mixing for $\Delta m^{2} \gtrsim 10^{-3} \mathrm{eV}^{2}$ [33].

For convenience, in the discussion we use the standard SMA, LMA, LOW and VO names for the regions in the $\tan ^{2} \vartheta-\Delta m^{2}$ plane. We consider these regions as enlarged with respect to the usual ones (see [25]), because the model independent credible regions that we obtain are slightly larger than the corresponding model dependent ones: Small Mixing Angle (SMA) for

$$
\tan ^{2} \vartheta \lesssim 10^{-2}, \quad 10^{-8} \mathrm{eV}^{2} \lesssim \Delta m^{2} \lesssim 10^{-3} \mathrm{eV}^{2}
$$

Large Mixing Angle (LMA) for

$$
10^{-2} \lesssim \tan ^{2} \vartheta \lesssim 10^{2}, \quad 3 \times 10^{-6} \mathrm{eV}^{2} \lesssim \Delta m^{2} \lesssim 10^{-3} \mathrm{eV}^{2},
$$

LOW for

$$
10^{-2} \lesssim \tan ^{2} \vartheta \lesssim 10^{2}, \quad 10^{-8} \mathrm{eV}^{2} \lesssim \Delta m^{2} \lesssim 3 \times 10^{-6} \mathrm{eV}^{2}
$$

\footnotetext{
3 Credible regions, also known as highest posterior density regions, contain a specified fraction of the posterior probability and all values
} of the parameters inside the credible regions have higher probability density than those outside (see, for example, Ref. [38]). 
Vacuum Oscillation (VO) for

$$
10^{-2} \lesssim \tan ^{2} \vartheta \lesssim 10^{2}, \quad \Delta m^{2} \lesssim 10^{-8} \mathrm{eV}^{2} .
$$

The plan of this paper is as follows. In Section [I] we present the sampling distributions in the two types of analysis that we perform: the Rates Analysis of Homestake, GALLEX+GNO+SAGE, SNO and Super-Kamiokande total rates and the Global Analysis of Homestake, GALLEX+GNO+SAGE and SNO rates and Super-Kamiokande day and night spectra. In Section III we present the prior distributions for the cross sections in the Rates and Global Analyses. In Section IV we perform a SSM model dependent analysis in order to check the validity of our approach by comparing the results with those obtained with a standard Bayesian analysis in Ref. [25]. In Section V we present and discuss the results of the model independent analysis of solar neutrino data. In the concluding Section $\mathrm{VI}$ we summarize our results.

\section{SAMPLING DISTRIBUTION}

Following the tradition, we perform two types of analysis, with different data sets. In the Rates Analysis (Subsection II A) we consider the total rates measured by solar neutrino experiments (Homestake, GALLEX+GNO+SAGE, SNO, Super-Kamiokande). In the Global Analysis (Subsection IIB) we consider the total rates of the Homestake, GALLEX+GNO+SAGE, SNO experiments and the Super-Kamiokande day and night electron energy spectra. Both analyses take into account also the data of the CHOOZ experiment 33].

Since the solar and CHOOZ data are independent, the sampling probability distribution can be written as

$$
p\left(\mathrm{D} \mid \tan ^{2} \vartheta, \Delta m^{2}, \Phi, \mathbf{C}, \mathrm{I}\right)=p\left(\mathrm{D}_{\mathrm{S}} \mid \tan ^{2} \vartheta, \Delta m^{2}, \Phi, \mathbf{C}, \mathrm{I}\right) p\left(\mathrm{D}_{\mathrm{C}} \mid \tan ^{2} \vartheta, \Delta m^{2}, \mathrm{I}\right),
$$

where $\mathrm{D}_{\mathrm{S}}$ represents the solar data and $\mathrm{D}_{\mathrm{C}}$ represents the positron spectra measured in the CHOOZ experiment, that are obviously independent from the solar neutrino fluxes and detection cross sections.

Assuming a normal distribution of experimental errors, the sampling probability distribution of $\mathrm{CHOOZ}$ data is given by

$$
p\left(\mathrm{D}_{\mathrm{C}} \mid \tan ^{2} \vartheta, \Delta m^{2}, \mathrm{I}\right)=\int \mathrm{d} \alpha_{\mathrm{C}} \frac{e^{-X_{\mathrm{C}}^{2} / 2}}{(2 \pi)^{N_{\mathrm{C}} / 2} \sqrt{\left|V_{\mathrm{C}}\right|}},
$$

where we have marginalized over the nuisance parameter $\alpha_{\mathrm{C}}$, that is the absolute normalization constant of CHOOZ positron spectra 33 . Here $N_{\mathrm{C}}=14$ is the number of CHOOZ data points, $X_{\mathrm{C}}^{2}$ is the CHOOZ least-squares function and $V_{\mathrm{C}}$ is the corresponding covariance matrix of uncertainties.

The CHOOZ least-squares function $X_{\mathrm{C}}^{2}$ is calculated as in the analysis A of Ref. [33], with the following approximations. Since we do not know the antineutrino spectrum, the spatial distribution functions of the reactor cores and detector and the detector response function linking the real and visible positron energies, for each energy bin we calculated the oscillation probability at the average energy of the bin and at the average distance of the detector from each of the two reactors [47. This approximation is quite good because we are interested in small values of $\Delta m^{2}$, for which the energy and distance dependence of the survival probability of the $\bar{\nu}_{e}$ 's in the CHOOZ experiment is very weak. We calculate $X_{\mathrm{C}}^{2}$ as in Eq. (13) of Ref. [33, with the only difference that we neglect the energy-scale calibration factor, whose small uncertainty $(1.1 \%)$ is practically negligible:

$$
X_{\mathrm{C}}^{2}=\sum_{j_{1}, j_{2}=1}^{N_{\mathrm{C}}}\left(R_{j_{1}}^{(\mathrm{th})}-\alpha_{\mathrm{C}} R_{j_{1}}^{(\mathrm{ex})}\right)\left(V_{\mathrm{C}}^{-1}\right)_{j_{1} j_{2}}\left(R_{j_{2}}^{(\mathrm{th})}-\alpha_{\mathrm{C}} R_{j_{2}}^{(\mathrm{ex})}\right)+\left(\frac{\alpha_{\mathrm{C}}-1}{\sigma_{\alpha_{\mathrm{C}}}}\right)^{2},
$$

where $\sigma_{\alpha_{\mathrm{C}}}=2.7 \times 10^{-2}$ is the uncertainty of the absolute normalization constant $\alpha_{\mathrm{C}}$ [33]. We calculate the CHOOZ covariance matrix $V_{\mathrm{C}}$ as described in Eq. (12) of Ref. [33. The only missing information in Ref. [33] is the value of the systematic uncertainties of the positron energy bins, for which only the values for positron energy 2 and $6 \mathrm{MeV}$ are given. For the other bins we take systematic uncertainties interpolated linearly between these two values.

\section{A. Rates Analysis}

Assuming a normal distribution of experimental errors, the sampling probability distribution of solar data in the Rates Analysis is given by

$$
p\left(\mathrm{D}_{\mathrm{S}} \mid \tan ^{2} \vartheta, \Delta m^{2}, \Phi, \mathbf{C}, \mathrm{I}\right)=\frac{e^{-X_{\mathrm{S}}^{2} / 2}}{(2 \pi)^{N_{\mathrm{S}} / 2} \sqrt{\left|V_{\mathrm{S}}\right|}},
$$


where $N_{\mathrm{S}}=4$ is the number of solar data points. $X_{\mathrm{S}}^{2}$ is the solar least-squares function and $V_{\mathrm{S}}$ is the corresponding covariance matrix.

The solar least squares function $X_{\mathrm{S}}^{2}$ is given by

$$
X_{\mathrm{S}}^{2}=\sum_{j=1}^{N_{\mathrm{S}}} \frac{\left(R_{j}^{(\mathrm{ex})}-R_{j}^{(\mathrm{th})}\right)^{2}}{\sigma_{j}^{2}}
$$

where $R_{j}^{(\mathrm{ex})}$ is the event rate measured in the $j^{\text {th }}$ experiment and $R_{j}^{(\mathrm{th})}$ is the corresponding theoretical event rate, that depends on $\Delta m^{2}, \tan ^{2} \theta$, the neutrino fluxes and the neutrino interaction cross sections in the detectors. The index $j=1, \ldots, 4$ indicate the four types of solar neutrino experiments listed in Table I together with the corresponding event rates and experimental uncertainties $\sigma_{j}$, calculated by adding in quadrature the statistical and systematic uncertainties for each experiment.

Since the uncertainties of the rates of different experiments are uncorrelated, the determinant $\left|V_{\mathrm{S}}\right|$ of the solar covariance matrix in Eq. (14) is simply given by

$$
\left|V_{\mathrm{S}}\right|=\prod_{j=1}^{N_{\mathrm{S}}} \sigma_{j}^{2}
$$

The theoretical event rate $R_{j}^{(\mathrm{th})}$ can be written as

$$
R_{j}^{(\mathrm{th})}=\sum_{i=1}^{8} R_{i j}^{(\mathrm{th})}
$$

where

$$
R_{i j}^{(\mathrm{th})}=\Phi_{i} C_{i j} P_{i j}\left(\Delta m^{2}, \tan ^{2} \theta\right)
$$

is the theoretical event rate in the $j^{\text {th }}$ experiment due to the neutrino flux $\Phi_{i}$ produced in the $i^{\text {th }}$ thermonuclear reaction of the $p p$ and $\mathrm{CNO}$ cycles in the sun $\left(i=1, \ldots, 8\right.$ for the $p p, p e p, h e p,{ }^{7} \mathrm{Be},{ }^{8} \mathrm{~B},{ }^{13} \mathrm{~N},{ }^{15} \mathrm{O},{ }^{17} \mathrm{~F}$ thermonuclear reactions, respectively). $C_{i j}$ is the corresponding energy-averaged cross section and $P_{i j}\left(\Delta m^{2}, \tan ^{2} \theta\right)$ is the corresponding averaged survival probability of solar $\nu_{e}$ 's, that depends on $\Delta m^{2}$ and $\tan ^{2} \theta$ (in the case of the Super-Kamiokande experiment, $j=4$, also the averaged $\nu_{e} \rightarrow \nu_{a}$ transition probability must be properly taken into account).

For the calculation of the probabilities $P_{i j}\left(\Delta m^{2}, \tan ^{2} \theta\right)$ we have used the tables of neutrino spectra, solar density and radiochemical detector cross sections available in Bahcall's web pages [48], BP2000 Standard Solar Model [11]. For the calculation of the theoretical rate of the SNO experiment we used the charged-current cross section given in Refs. [49, 50, 51]. We did not consider the contribution of radiative corrections discussed in Refs. [52, 53], which increase the neutrino-deuteron cross section by a few percents, that is smaller than the experimental uncertainty of the SNO event rate [2, 54].

The probability of neutrino oscillations is calculated with an unified approach that allows to pass continuously from the vacuum oscillation regime to MSW transitions [44, 45] through the quasi-vacuum regime [55, 56], using the quasivacuum analytical prescription given in Ref. [57] (see also Refs. [58, 59]), the usual prescription for the MSW survival probability (see [56, 60]) and the level crossing probability appropriate for an exponential density profile [61, 62]. We calculate the regeneration in the Earth using a two-step model of the Earth density profile [63, 64, 65, 66, 67, that is known to produce results that do not differ appreciably from those obtained with a less approximate density profile.

\section{B. Global Analysis}

In the Global Analysis, instead of the total rate of the Super-Kamiokande experiment we consider the data on the Super-Kamiokande day and night electron energy spectra presented in Ref. [68], that contain information on the total rate plus the shape of the energy spectrum. Assuming a normal distribution of experimental errors, the sampling probability distribution of solar data in the Global Analysis is given by

$$
p\left(\mathrm{D}_{\mathrm{S}} \mid \tan ^{2} \vartheta, \Delta m^{2}, \Phi, \mathbf{C}, \mathrm{I}\right)=\frac{e^{-X_{\mathrm{S}}^{2} / 2}}{(2 \pi)^{N_{\mathrm{S}} / 2} \sqrt{\left|V_{\mathrm{S}}\right|}} \frac{e^{-X_{\mathrm{SK}}^{2} / 2}}{(2 \pi)^{N_{\mathrm{SK}} / 2} \sqrt{\left|V_{\mathrm{SK}}\right|}},
$$


where the first factor takes into account the rates measured in the Homestake, GALLEX+GNO+SAGE and SNO experiments. Therefore, the first factor in Eq. (19) is calculated as in the Rates Analysis, with the only difference that $N_{\mathrm{S}}=3$.

The second factor in Eq. (19) takes into account the Super-Kamiokande data on the day and night electron energy spectra.

The Super-Kamiokande least-squares function $X_{\mathrm{SK}}^{2}$ is given by

$$
X_{\mathrm{SK}}^{2}=\sum_{b_{1}, b_{2}=1}^{38}\left(R_{b_{1}}^{(\mathrm{ex})}-R_{b_{1}}^{(\mathrm{th})}\right)\left(V_{\mathrm{SK}}^{-1}\right)_{b_{1} b_{2}}\left(R_{b_{2}}^{(\mathrm{ex})}-R_{b_{2}}^{(\mathrm{th})}\right),
$$

with the indexes $b_{1}, b_{2}$ run from 1 to 38 for the bins of the Super-Kamiokande day and night electron energy spectra given in Table III of Ref. [68]. The indexes $b_{1}, b_{2}=1, \ldots, 19$ refer to the day spectrum and the indexes $b_{1}, b_{2}=20, \ldots, 38$ refer to the night spectrum. The covariance matrix $V_{\mathrm{SK}}$ is written as

$$
\left(V_{\mathrm{SK}}\right)_{b_{1} b_{2}}=\delta_{b_{1} b_{2}}\left[\left(\sigma^{(\mathrm{sta})}\right)_{b_{1}}^{2}+\left(\sigma_{\mathrm{unc}}^{(\mathrm{sys})}\right)_{b_{1}}^{2}\right]+\left(\sigma_{\mathrm{cor}}^{(\mathrm{sys})}\right)_{b_{1}}\left(\sigma_{\mathrm{cor}}^{(\mathrm{sys})}\right)_{b_{2}}+\left(\sigma_{\mathrm{unc}}^{(\mathrm{sys})}\right)_{b_{1}}^{2}\left[\delta_{b_{1}\left(b_{2}-19\right)}+\delta_{b_{1}\left(b_{2}+19\right)}\right] .
$$

The statistical uncertainties $\left(\sigma^{\text {(sta) }}\right)_{b}$ are given in the third (for $b=1, \ldots, 19$, day spectrum) and fourth (for $b=$ $20, \ldots, 38$, night spectrum) columns of Table III in Ref. [68]. The experimental uncorrelated systematic uncertainties $\left(\sigma_{\text {unc }}^{\text {(sys) }}\right)_{b}$ (for $\left.b=1, \ldots, 38\right)$ are given by $R_{b}^{(\text {ex })} \delta_{b, \text { unc }}^{(\text {ex })}$, with $R_{b}^{(\text {ex })}$ listed in the third (for $b=1, \ldots, 19$, day spectrum) and fourth (for $b=20, \ldots, 38$, night spectrum) columns of Table III in Ref. [68]. The last term in Eq. (21) is due to the assumption of a full correlation of the systematic uncertainties of the day and night bins with the same energy. The values of $\delta_{b, \text { unc }}^{(\text {ex })}=\delta_{b+19 \text {, unc }}^{(\mathrm{ex})}$ for $b=1, \ldots, 19$ are listed in the sixth column of Table III in Ref. [68] (we took the bigger between the positive and negative values).

The experimental correlated systematic uncertainties are given by $\left(\sigma_{\mathrm{cor}}^{(\mathrm{sys})}\right)_{b}=R_{b}^{(\mathrm{ex})} \delta_{b, \mathrm{cor}}^{\text {(sys) }}$. Unfortunately, the relative correlated systematic uncertainties presented by the Super-Kamiokande collaboration in the fifth column of Table III in Ref. [68] contain the contribution from the energy-dependent part of the theoretical uncertainty on the ${ }^{8} \mathrm{~B}$ neutrino spectrum given in Ref. [69], that is taken into account by the prior probability distribution discussed in Subsection IIIB. We list in Table II the values of relative theoretical uncertainty $\delta_{b, \mathrm{cor}}^{(\mathrm{th})}$ for the rates in the SuperKamiokande spectral bins $(b=1, \ldots, 38)$ due to the theoretical uncertainty on the ${ }^{8} \mathrm{~B}$ neutrino spectrum extracted from Fig. 5 of Ref. [69]. In order to avoid double-counting, we subtracted quadratically the energy-dependent part of $\delta_{b, \text { cor }}^{(\text {th })}$ (i.e. $\delta_{b, \text { cor }}^{(\text {th })}-\delta_{1, \text { cor }}^{(\text {th })}$, where $b=1$ is the index of the first Super-Kamiokande energy bin) from the relative correlated systematic uncertainties in the fifth column of Table III in Ref. [68] (for which we took the bigger between the positive and negative values). The resulting values of $\delta_{b, \mathrm{cor}}^{(\mathrm{sys})}$ are listed in Table $\amalg$.

\section{CROSS SECTIONS}

In this section we discuss the prior distribution $p(\mathbf{C} \mid \mathrm{I})$ for the energy-averaged cross sections of neutrino interaction in the detectors. In the case of the Super-Kamiokande experiment there is practically no uncertainty on the value of the neutrino-electron elastic cross section, but there is an uncertainty of the shape of the ${ }^{8} \mathrm{~B}$ neutrino spectrum [69, 70]. We take this uncertainty into account both in the Rates Analysis and Global Analysis, as described in the following subsections.

\section{A. Rates Analysis}

In the Rates Analysis the number of experimental data points is $N_{\mathrm{S}}=4$ (see Table $\mathbb{\text { I) }}$ ), leading to a $8 \times 4$ matrix $\mathbf{C}$ of energy averaged cross sections.

Assuming a complete correlation of the errors of the energy-averaged cross sections of different neutrino fluxes in each experiment [71, the cross section $C_{i j}$ of neutrino detection in the $j^{\text {th }}$ experiment averaged over the energy spectrum of the $i^{\text {th }}$ neutrino flux can be written as

$$
C_{i j}=\left(1+\xi_{j} \Delta \ln C_{i j}\right) C_{i j}^{0},
$$

where $C_{i j}^{0}$ is the standard averaged cross section, $\Delta \ln C_{i j}$ is the relative uncertainty of $C_{i j}^{0}$, and $\xi_{j}$ is an unknown parameter that quantifies the deviation of the averaged cross section from its standard value. Assuming a normal 
distribution of errors, for each $\xi_{j}$ we adopt the prior distribution

$$
p\left(\xi_{j} \mid \mathrm{I}\right)=\frac{e^{-\xi_{j}^{2} / 2}}{\sqrt{2 \pi}} .
$$

The prior distributions for different $\xi_{j}$ 's are independent because the theoretical errors of the energy-averaged cross sections of neutrino interaction in different experiments are uncorrelated. The prior distribution $p(\mathbf{C} \mid \mathrm{I})$ of energy averaged cross section is obtained from Eqs. (22) and (23). In practice, since all quantities that we calculate are integrated over $\mathrm{d} \mathbf{C}$ using a Monte Carlo, the prior distribution $p(\mathbf{C} \mid \mathrm{I})$ is generated by the Monte Carlo from the prior distribution (23) of $\xi_{j}$, using Eq. (22).

This procedure may seem arbitrarily complicated, but one must notice that it is not possible to write a multi-normal distribution for fully correlated quantities as $C_{i j}$ for $i=1, \ldots, 8$, because the covariance matrix would be singular. Indeed, complete correlation means that the errors are not independent: the deviation of one $C_{i j}$ with respect to $C_{i j}^{0}$ determines the deviations of all the others $C_{i^{\prime} j}$ from $C_{i^{\prime} j}^{0}$, with $i^{\prime} \neq i$, as quantified in Eq. (22).

The values of $C_{i j}^{0}$ that we use for the Chlorine $(j=1)$ and Gallium $(j=2)$ experiments are given in Table V of Ref. [32]. For the SNO experiment $(j=3)$ with kinetic energy threshold $T_{e}^{\mathrm{SNO}}=6.75 \mathrm{MeV}$ for the detected electrons, we calculated

$$
C_{33}^{0}=1.82 \times 10^{-7} \mathrm{~cm}^{2} \mathrm{~s}, \quad C_{53}^{0}=1.98 \times 10^{-7} \mathrm{~cm}^{2} \mathrm{~s},
$$

and all the other $C_{i 3}^{0}$ are zero. For the Super-Kamiokande experiment $(j=4)$ with kinetic energy threshold $T_{e}^{\mathrm{SK}}=$ $4.50 \mathrm{MeV}$ for the detected electrons, we calculated

$$
C_{34}^{0}=1.04 \times 10^{-7} \mathrm{~cm}^{2} \mathrm{~s}, \quad C_{54}^{0}=1.98 \times 10^{-7} \mathrm{~cm}^{2} \mathrm{~s},
$$

and all the other $C_{i 4}^{0}$ are zero.

The values of the relative uncertainties $\Delta \ln C_{i j}=\Delta C_{i j} / C_{i j}$ for $j=1,2\left({ }^{37} \mathrm{Cl},{ }^{71} \mathrm{Ga}\right.$ experiments $)$ are given in Table VI of Ref. [32]. For the SNO experiment $(j=3), \Delta \ln C_{33}=\Delta \ln C_{53}=3.0 \times 10^{-2}$ [2, 49, 51] and all the other $\Delta \ln C_{i 3}$ are zero. For the Super-Kamiokande experiment $(j=4)$ we take $\Delta \ln C_{54}=2.0 \times 10^{-2}$ in order to take into account the uncertainty of the shape of the ${ }^{8} \mathrm{~B}$ neutrino spectrum calculated in Ref. [69]. All the other $\Delta \ln C_{i 4}$ are zero.

\section{B. Global Analysis}

In the Global Analysis the prior distribution of the averaged cross sections in the Chlorine $(j=1)$, Gallium $(j=2)$ and SNO $(j=3)$ experiments is the same as in the Rates Analysis. For the Super-Kamiokande experiment instead of the total rate, we consider the data on the 38 bins of the Super-Kamiokande day and night electron energy spectra (see Table II). Hence, the number of experimental data points in the Global Analysis is $N_{\mathrm{S}}=41$, leading to a $8 \times 41$ matrix $\mathbf{C}$ of energy averaged cross sections.

The energy-averaged cross sections $C_{i j}$ for $j \leq 3$ are given by Eq. (22), with the distribution of the normalized deviations $\xi_{j}$ given in Eq. (23).

The energy-averaged cross sections $C_{5 j}$, with $j=4, \ldots, 41$, of ${ }^{8} \mathrm{~B}$ neutrino detection for the Super-Kamiokande day and night spectral bins that take into account the fully correlated uncertainty due to the uncertainty of the shape of the ${ }^{8} \mathrm{~B}$ neutrino spectrum [69, 70] are given by

$$
C_{5(b+3)}=\left(1+\xi_{4} \delta_{b, \mathrm{cor}}^{(\mathrm{th})}\right) C_{5(b+3)}^{0} \quad(b=1, \ldots, 38),
$$

with the normalized deviation $\xi_{4}$ distributed as $p\left(\xi_{4} \mid \mathrm{I}\right)$ given in Eq. (23) with $j=4$. In Eq. (26), $C_{5(b+3)}^{0}$ is the standard energy-averaged cross section of ${ }^{8} \mathrm{~B}$ neutrino detection in the $b^{\text {th }}$ Super-Kamiokande bin given in Table II, together with $C_{3(b+3)}^{0}$, relative to the hep neutrino flux (we take $C_{3(b+3)}=C_{3(b+3)}^{0}$ and all the other $C_{i(b+3)}=0$ ). The values of relative theoretical uncertainty $\delta_{b, \text { cor }}^{(\text {th) }}$ given in Fig. 5 of Ref. 69. for the rates in the Super-Kamiokande spectral bins $(b=1, \ldots, 38)$ due to the theoretical uncertainty on the ${ }^{8} \mathrm{~B}$ neutrino spectrum are listed in Table [I].

\section{MODEL DEPENDENT ANALYSIS}

In this Section we perform a Bayesian model dependent analysis of solar neutrino data assuming the solar neutrino fluxes predicted by the BP2000 Standard Solar Model [11]. In this analysis we take into account of all the theoretical 
uncertainties through the prior distributions. We check the validity of this approach comparing the results with those obtained with a standard Bayesian analysis in Ref. [25].

The sampling distributions and the prior distributions of detection cross sections are described, respectively, in Sections II and III. In the following Subsection IVA we describe the SSM prior distribution of fluxes used in the model dependent analysis. In Section IVB we discuss our results for the calculation of the Bayesian credible regions in the plane of the oscillation parameters $\tan ^{2} \vartheta$ and $\Delta m^{2}$, to be compared with the corresponding ones presented in Ref. 25.

\section{A. SSM Prior Distribution of Fluxes}

In the model dependent analysis we assume a multi-normal prior distribution for the neutrino fluxes centered on the BP2000 SSM values of the neutrino fluxes, $\Phi_{i}^{\mathrm{SSM}}$ [1], with the standard correlated uncertainties (see [32, 72]):

$$
p(\Phi \mid \mathrm{I})=N \exp \left[-\frac{1}{2} \sum_{i_{1}, i_{2}=1}^{8}\left(\Phi_{i_{1}}-\Phi_{i_{1}}^{\mathrm{SSM}}\right)\left(V_{\Phi}^{-1}\right)_{i_{1} i_{2}}\left(\Phi_{i_{2}}-\Phi_{i_{2}}^{\mathrm{SSM}}\right)\right] \prod_{i=1}^{8} \theta\left(\Phi_{i}\right),
$$

where $N$ is a normalization constant and $\theta\left(\Phi_{i}\right)=1$ for $\Phi_{i} \geq 0$ and $\theta\left(\Phi_{i}\right)=0$ for $\Phi_{i}<0$. $V_{\Phi}$ is the covariance matrix of the fluxes, given by [32, 72]

$$
\left(V_{\Phi}\right)_{i_{1} i_{2}}=\Phi_{i_{1}}^{\mathrm{SSM}} \Phi_{i_{2}}^{\mathrm{SSM}} \sum_{k=1}^{12} \alpha_{i_{1}, k} \alpha_{i_{2}, k}\left(\Delta \ln X_{k}\right)^{2}
$$

Here $X_{k}$, with $k=1, \ldots, 12$, are the twelve input astrophysical parameters in the SSM $\left(S_{1,1}, S_{3,3}, S_{3,4}, S_{1,14}, S_{1,7}\right.$, Luminosity, $Z / X$, Age, Opacity, Diffusion, $\left.C_{7 \text { Be }}, S_{1,16}\right)$, whose relative uncertainties $\Delta \ln X_{k}$ determine the correlated uncertainties of the neutrino fluxes $\Phi_{i}^{S S M}$ through the logarithmic derivatives

$$
\alpha_{i, k}=\frac{\partial \ln \Phi_{i}^{\mathrm{SSM}}}{\partial \ln X_{k}}
$$

We adopt the values of $\alpha_{i, k}$ and $\Delta \ln X_{k}$ given in Ref. [32], except for $\Delta \ln X_{7}$ (relative to $Z / X$ ), whose value has been updated in the BP2000 SSM [1] from 0.033 to 0.061 , and $\alpha_{i, 12}=\delta_{i 8}, \Delta \ln X_{12}=0.181$, that have been introduced for the first time in the BP2000 SSM [11]. $X_{12}$ is the astrophysical factor $S_{1,16}$ for the reaction ${ }^{16} \mathrm{O}(p, \gamma){ }^{17} \mathrm{~F}$ that determines the small ${ }^{17} \mathrm{~F}$ neutrino flux $(i=8)$.

\section{B. Credible Regions}

The credible regions with $90 \%, 95 \%$ and $99 \%$ posterior probability calculated through Eq. (5) are shown in Fig. 1 for the Rates Analysis and in Fig. 2 for the Global Analysis. One can see that they are almost identical to the corresponding regions in Figs. 3 and 4 of Ref. [25], obtained with the standard method, in which the uncertainties of the fluxes and cross sections are taken into account in the covariance matrix that determines the likelihood function.

The integral probabilities of the LMA, VO, SMA and LOW regions presented in Table III almost coincide with those obtained in Ref. [25. As noted in Ref. 25], these probabilities favor the LMA solution of the solar neutrino problem.

Table III shows also the values of the integral probabilities of large and small values of $\tan ^{2} \vartheta$ and $\Delta m^{2}$, that favor large mixing and large values of $\Delta m^{2}$.

In conclusion of this section, we have shown that in the model dependent analysis of solar neutrino data the new approach that we follow here, in which all the theoretical uncertainties are taken into accounts in the priors, gives approximately the same results as the standard one followed in Ref. [25], in which the theoretical uncertainties are taken into account in the covariance matrix that determines the likelihood function. Therefore, we conclude that the new approach is compatible with the standard one and we can apply it to the model independent analysis of solar neutrino data.

Let us notice, however, that the new approach is not convenient for the model dependent analysis of solar neutrino data, because it needs the integrations over the neutrino fluxes and cross sections in Eq. (6). 


\section{MODEL INDEPENDENT ANALYSIS}

This is the main Section of the paper, in which we present the results of the model independent analysis of solar neutrino data assuming a flat prior probability distribution of neutrino fluxes, that is explained in the following Subsection $\mathrm{VA}$.

In Subsection $\mathrm{VB}$ we present the model independent allowed ranges for the neutrino mixing parameters tan ${ }^{2} \vartheta$, $\Delta m^{2}$. In Subsection $\mathrm{VG}$ we discuss the information on the solar neutrino fluxes obtained from the model independent posterior distribution. Finally, in Subsection VD we compare the probabilities of the hypotheses of no neutrino oscillations and neutrino oscillations.

\section{A. Model Independent Prior Distribution of Fluxes}

The Standard Solar Model has reached a reasonable degree of credibility in recent years as a consequence of its very good agreement with helioseismological measurements [11, 73]. Therefore, we think that the true values of the neutrino fluxes cannot be too different from those of the SSM.

In our model independent analysis we assume a flat prior distribution from zero to twice the BP2000 SSM value 11] for all the neutrino fluxes except hep and $p p$.

Since the astrophysical $S$-factor of the hep reaction is very difficult to calculate (see Ref. 74 ), the value of the hep flux is the most uncertain one and no uncertainty is given in the BP2000 SSM. However, the Super-Kamiokande Collaboration measured [1] a 90\% CL upper limit for the hep neutrino flux of 4.3 times the BP2000 SSM prediction. Hence, in the model independent analysis we take for the hep flux a flat prior distribution from zero to ten times the BP2000 SSM value [11].

The prior distribution of the $p p$ flux is obtained from the prior distributions of the other fluxes and the luminosity constraint (see Ref. 75)

$$
\sum_{i} \alpha_{i} \Phi_{i}=K_{\odot}
$$

where $\alpha_{i}$ is the average thermal energy released together with a neutrino from the source $i$ given in Ref. 750 and $K_{\odot}=\mathcal{L}_{\odot} / 4 \pi R^{2}=8.527 \times 10^{11} \mathrm{MeV} \mathrm{cm}^{-2} \mathrm{~s}^{-1}$ is the solar constant $\left(\mathcal{L}_{\odot}=2.398 \times 10^{39} \mathrm{MeV} \mathrm{s}^{-1}\right.$ is the luminosity of the sun and $R=1.496 \times 10^{13} \mathrm{~cm}$ is the sun-earth distance). The choice of the $p p$ flux as the dependent flux to be determined by the luminosity constraint (30) is mandatory, because the $p p$ flux, being the largest one in the SSM, is the only flux that can exhaust by itself the luminosity constraint without assuming an unreasonably large value.

The resulting prior distribution of the ratio $\Phi_{p p} / \Phi_{p p}^{\mathrm{SSM}}$ is almost flat in the range $[0.904,1.094]$, as can be seen from Figs. 15 and 16 (thick dashed curve). The upper value of this range corresponds to the maximum $p p$ flux allowed by the luminosity constraint (30). The lower value depends on the choice of the range of the prior distributions of the other fluxes. As shown in Figs. 15 and 16, the prior distribution of the $p p$ flux is almost exactly symmetric with respect to $\Phi_{p p} / \Phi_{p p}^{\mathrm{SSM}}=1$ and is exactly flat in the range $[0.94,1.06]$ (the small fluctuations of the prior distribution in Figs. 15 and 16 is due to the fact that the prior distributions of the neutrino fluxes are generated through a Monte Carlo, in order to perform the integrals over the neutrino fluxes in Eq. (6)).

In Table [V] we list the relative prior uncertainties $\Delta \Phi_{i} / \Phi_{i}^{\mathrm{SSM}}$ of the neutrino fluxes in the model independent analysis and we confront them with the relative uncertainties $\left(\Delta \Phi_{i}^{\mathrm{SSM}} / \Phi_{i}^{\mathrm{SSM}}\right)_{(1 \sigma)}$ in the BP2000 Standard Solar Model and with the relative differences between the fluxes predicted by several old and new models and the BP2000 SSM. One can see that the relative prior uncertainties in the model independent analysis are much larger than the relative uncertainties in the BP2000 Standard Solar Model and they are also much larger than the relative differences between the fluxes predicted by other models and the BP2000 SSM. Therefore, our choice of prior distributions for the neutrino fluxes is indeed appropriate for a model independent analysis of solar neutrino data.

\section{B. Oscillation Parameters}

In this Subsection we present our results for the model independent credible regions in the $\tan { }^{2} \vartheta-\Delta m^{2}$ plane obtained with the posterior distribution (6) marginalized with respect to the neutrino fluxes and cross sections.

The credible regions with $90 \%, 95 \%$ and $99 \%$ posterior probability obtained with the Rates Analysis are shown in Fig. 3. One can see that they are significantly larger than the model dependent credible regions in Fig. 1, but 
still restricted in the parameter space: a large area of the parameter space shown in Fig. 3 is excluded ${ }^{4}$ in a model independent way. In particular, the absence of neutrino oscillations, corresponding to $\left(\Delta m^{2} \tan ^{2} \vartheta\right) \lesssim 10^{-12} \mathrm{eV}^{2}$, is excluded.

In Fig. 3 one can distinguish clearly the $90 \%$ and $95 \%$ allowed LMA, SMA, LOW and VO regions that are obviously larger than the corresponding regions obtained in the model dependent analysis (Fig. 1). The 99\% allowed regions are less defined, but large values of $\Delta m^{2}$, corresponding to the LMA and SMA regions with $\Delta m^{2} \gtrsim 3 \times 10^{-6} \mathrm{eV}^{2}$, are separated from small values of $\Delta m^{2}$, corresponding to the LOW and VO regions with $\Delta m^{2} \lesssim 3 \times 10^{-6} \mathrm{eV}^{2}$. The LMA and SMA $99 \%$ allowed regions are connected through a channel at $\Delta m^{2} \simeq 1.3 \times 10^{-4} \mathrm{eV}^{2}$. The LOW $99 \%$ region is connected with the upper VO $99 \%$ region, but there are still two separated VO $99 \%$ regions at $\Delta m^{2} \simeq 3 \times 10^{-10} \mathrm{eV}^{2}$ and $\Delta m^{2} \simeq 1 \times 10^{-10} \mathrm{eV}^{2}$.

All experiments are important in order to restrict the model independent allowed regions, as one can see confronting Fig. 3 with the four figures 14 , each one obtained neglecting the data of one of the four experiments in Table 1 . In particular, one can see that the Gallium data of GALLEX+GNO+SAGE and the rate measured in the SuperKamiokande experiment are crucial in order to separate the 90\% LMA and LOW regions, and all experiment are important for the separation of the $95 \%$ and $99 \%$ LMA and LOW regions. The SNO and Super-Kamiokande rates are important for the exclusion of a large part of the "dark-side" region $\left(\tan ^{2} \vartheta>1\right)$.

Figure 8 illustrates the quality of the fit of solar neutrino data in the Rates Analysis for some selected values of $\tan ^{2} \vartheta$ and $\Delta m^{2}$. The corresponding best-fit values of the neutrino fluxes, normalized to the BP2000 SSM prediction, are given in Table $\mathrm{V}$.

The solid, long-dashed, short-dashed and dotted lines in Fig. 8A correspond, respectively, to the best-fit points in the SMA, LMA, LOW and VO regions (we call "best-fit point" the point with highest posterior density of probability). From Fig. 8A one can see that the best-fit LMA solution fits very well the data, the best-fit SMA solution fits well the data, but it is slightly off the SNO rate, the best-fit VO solution do not fit well the Gallium rate, and the best-fit LOW solution fit of the Gallium and Chlorine data is rather bad.

The solid line in Fig. $8 \mathrm{~B}$ corresponds to $\tan ^{2} \vartheta=1.0 \times 10^{-2}$ and $\Delta m^{2}=3.0 \times 10^{-5} \mathrm{eV}^{2}$, a point near the center of a quasi-triangular excluded region in Figs. 37. From Fig. $8 \mathrm{~B}$ one can see that in this case the energy dependence of the oscillation probability is very strong, typical of small mixing angle MSW transitions (see [13, 14, 15, 45]), with small suppression of low-energy $p p$ neutrinos that do not cross the resonance and a large suppression of intermediate and high energy neutrinos. The fit of the Super-Kamiokande rate is less disastrous than the fit of the SNO rate because of the high flux of $\nu_{\mu, \tau}$ due to a large ${ }^{8} \mathrm{~B}$ flux at the limit of the maximum value $\Phi_{8 \mathrm{~B}} / \Phi_{8 \mathrm{~B}}^{\mathrm{SSM}}=2$, as shown in Table V. From Fig. $8 \mathrm{~B}$ one can infer that the quasi-triangular excluded region centered at $\tan ^{2} \vartheta \simeq 1.0 \times 10^{-2}$ and $\Delta m^{2} \simeq 3.0 \times 10^{-5} \mathrm{eV}^{2}$ is still excluded eliminating one data point, in agreement with Figs. 17 7.

The long-dashed line in Fig. $8 \mathrm{~B}$ corresponds to $\tan ^{2} \vartheta=5.0 \times 10^{-2}$ and $\Delta m^{2}=1.0 \times 10^{-6} \mathrm{eV}^{2}$, a point in a quasi-triangular excluded region at the center of Figs. 3 - One can see that in this region it is not possible to fit the Gallium, Chlorine and SNO data. The reason is a strong suppression of all energy neutrinos due to the crossing of the MSW resonance in the sun. Only the Super-Kamiokande rate can be fitted well taking a large ${ }^{8} \mathrm{~B}$ flux at the limit of the maximum value of the prior distribution, as shown in Table $\mathrm{V}$.

The short-dashed line in Fig. $8 \mathrm{~B}$ corresponds to $\tan ^{2} \vartheta=1.00$ and $\Delta m^{2}=9.0 \times 10^{-11} \mathrm{eV}^{2}$, a point near the center of an excluded area in the VO region in Figs. 3 .5 and 8. One can see that this region is excluded by the SNO rate, in agreement with the fact that it is not excluded in Fig. 6, obtained with the model independent Rates Analysis of Gallium, Chlorine and Super-Kamiokande data only.

The dotted line in Fig. $8 \mathrm{~B}$ corresponds to $\tan ^{2} \vartheta=1.00$ and $\Delta m^{2}=5.5 \times 10^{-12} \mathrm{eV}^{2}$, a point in the "Just So" region found in Ref. [76] in the SSM model dependent analysis of pre-SNO solar neutrino data. One can see that the SNO rate disfavors this region, that is indeed allowed in Fig. 6, obtained with the model independent Rates Analysis of Gallium, Chlorine and Super-Kamiokande data only.

Finally, the dash-dotted line in Fig. \&B corresponds to the case of no oscillations. Obviously, as shown in Table $\mathrm{V}$, in this case the flux of intermediate energy neutrinos must be suppressed and the ${ }^{8} \mathrm{~B}$ neutrino flux must have an intermediate value between those measured in the Super-Kamiokande 1] and SNO [2] experiments assuming no neutrino oscillations. The smaller experimental uncertainty of the Super-Kamiokande rate forces the ${ }^{8} \mathrm{~B}$ neutrino flux to be close to the one measured in the Super-Kamiokande experiment in the case of no-oscillations, leading to a bad fit of the SNO rate. This ${ }^{8} \mathrm{~B}$ neutrino flux adds to the $p p$ flux to give a rate in Gallium experiments that is too high, leading to a bad fit of Gallium data, as shown in Fig. 8 B.

Let us consider now the Global Analysis. Figure 9 shows the credible regions with 90\%, 95\% and $99 \%$ posterior

4 For convenience, we call "excluded" the areas outside of the credible regions, although they are not strictly speaking excluded, since they have a non-zero, albeit small, posterior probability. Similarly, we sometimes call "allowed" the areas within the credible regions. 
probability obtained with the Global Analysis. Again, the allowed regions are larger than those obtained with the model dependent analysis (see Fig. 2), but they are still clearly distinguishable and a large region of the parameter space is excluded. One can notice that in the model independent Global Analysis there is a very small SMA credible region at the $99 \%$ probability level, that is not present in the model dependent analysis.

In Fig. 9 the LMA regions with $90 \%, 95 \%$ and $99 \%$ probability are clearly separated from the other regions, whereas the LOW and VO credible regions are connected through the quasi-vacuum region at $\Delta m^{2} \sim 6 \times 10^{-9} \mathrm{eV}^{2}$. Several disconnected VO credible regions are present for $8 \times 10^{-11} \mathrm{eV}^{2} \lesssim \Delta m^{2} \lesssim 3 \times 10^{-9} \mathrm{eV}^{2}$.

Figure 10 illustrates the quality of the fit of solar neutrino data in the Global Analysis for some selected values of $\tan ^{2} \vartheta$ and $\Delta m^{2}$, with the corresponding best values of the neutrino fluxes normalized to the BP2000 SSM prediction given in Table VI.

The lines in Fig. 10A correspond to the same values of $\tan ^{2} \vartheta$ and $\Delta m^{2}$ as in Fig. \&A, i.e. to the best-fit point in the SMA, LMA, LOW and VO regions obtained in the Rates Analysis. The solid, long-dashed, short-dashed and dotted lines in Fig. 10B correspond, respectively, to the best-fit point in the SMA, LMA, LOW and VO regions obtained in the Global Analysis. The dash-dotted line in Fig. 10B corresponds to the case of no oscillations.

The best-fit SMA solution in the Rates Analysis (solid line in Fig. 10A) is disfavored by the Super-Kamiokande energy spectrum, because the corresponding electron-neutrino survival probability increases with energy too steeply. The best-fit SMA solution in the Global Analysis (solid line in Fig. 10B) has a smaller mixing angle, leading to a flatter energy dependence of the electron-neutrino survival probability, in agreement with the Super-Kamiokande spectrum. The survival probability of high-energy electron-neutrinos is close to one and the Super-Kamiokande data are fitted with a small ${ }^{8} \mathrm{~B}$ neutrino flux, as shown in Table $\mathrm{VI}\left(\Phi_{{ }_{\mathrm{B}}} / \Phi_{{ }_{\mathrm{B}}}^{\mathrm{SSM}} \simeq 0.53\right)$. In this case the theoretical rate for the SNO experiment $(j=3)$ is too high, as shown in Fig. 10 B, disfavoring the SMA solution.

Figure 10 shows that the data are well fitted in the LMA and LOW regions, whereas the VO solution is slightly disfavored by the highest-energy bins of the Super-Kamiokande spectrum. It is interesting to notice the difference of the LMA prediction for the day and night Super-Kamiokande energy bins, that is especially evident in Fig. 10A. This difference is due to the regeneration of electron neutrinos in their passage through the Earth.

The case of no oscillations (dash-dotted line in Fig. 10B) is compatible with the Super-Kamiokande energy spectrum through a small ${ }^{8} \mathrm{~B}$ neutrino flux, as shown in Table $\mathrm{VI}\left(\Phi_{{ }_{8} \mathrm{~B}} / \Phi_{8 \mathrm{~B}}^{\mathrm{SSM}} \simeq 0.44\right)$. However, this flux is too high to fit the Gallium $(j=1)$, Chlorine $(j=2)$ and $\operatorname{SNO}(j=3)$ rates.

Let us now determine the probabilities of the SMA, LMA, LOW and VO regions, that are given by the integrals of the posterior density over the appropriate ranges of the parameters given in Eqs. (17)-(10):

$$
p(\mathrm{R} \mid \mathrm{D}, \mathrm{I})=\int_{\mathrm{R}} \mathrm{d} \log \left(\tan ^{2} \vartheta\right) \operatorname{dlog}\left(\Delta m^{2}\right) p\left(\tan ^{2} \vartheta, \Delta m^{2} \mid \mathrm{D}, \mathrm{I}\right),
$$

with $\mathrm{R}=\mathrm{SMA}$, LMA, LOW, VO. The values of these probabilities in both the Rates Analysis and Global Analysis are listed in Table III, together with those obtained in the model dependent analysis presented in Section IV (see also 25]). One can see that the model independent and model dependent integral probabilities of the four regions are similar and the LMA region is favored in both cases, especially with the Global Analysis that, on the other hand, strongly disfavors the SMA region.

Bayesian Probability Theory allows also to calculate the separate posterior probability distributions for $\tan ^{2} \vartheta$ and $\Delta m^{2}$ through the marginalizations

$$
\begin{aligned}
& p\left(\tan ^{2} \vartheta \mid \mathrm{D}, \mathrm{I}\right)=\int \operatorname{dlog}\left(\Delta m^{2}\right) p\left(\tan ^{2} \vartheta, \Delta m^{2} \mid \mathrm{D}, \mathrm{I}\right), \\
& p\left(\Delta m^{2} \mid \mathrm{D}, \mathrm{I}\right)=\int \operatorname{dlog}\left(\tan ^{2} \vartheta\right) p\left(\tan ^{2} \vartheta, \Delta m^{2} \mid \mathrm{D}, \mathrm{I}\right) .
\end{aligned}
$$

Figures 11 and 12 show the model independent posterior distribution of $\tan ^{2} \vartheta$ in the Rates Analysis and Global Analysis, respectively. One can see that in both cases there is a wide and high peak at large mixing angles $\left(\tan ^{2} \vartheta \sim\right.$ 0.3). In the Rates Analysis there is also a small peak at small mixing angles $\left(\tan ^{2} \vartheta \sim 10^{-3}\right)$. The integral probabilities of large and small mixing are given in Table III, together with those obtained in the model dependent analysis of Section IV. One can see that the values are similar and both the model independent and the model dependent analyses favor large mixing $\left(0.1<\tan ^{2} \vartheta<10\right)$. This indication is very strong in both the model independent and the model dependent Global Analyses.

Small mixing is obtained either through small $(<0.1)$ or large $(>10)$ values of $\tan ^{2} \vartheta$, that correspond, respectively, to $\nu_{e} \simeq \nu_{1}$ and $\nu_{e} \simeq \nu_{2}$ (see Eq. (1)). Table III shows that both small and large values of $\tan ^{2} \vartheta$ are disfavored, especially large values, in what could be called "extreme dark side" $\left(\tan ^{2} \vartheta>10\right)$, following the "dark side" denomination of the region $\tan ^{2} \vartheta>1$ [77. 
Figures 13 and 14 show the posterior distribution of $\Delta m^{2}$ in the Rates Analysis and Global Analysis, respectively. The high and wide peak at $\Delta m^{2} \sim 10^{-5}-10^{-4} \mathrm{eV}^{2}$ in both figures implies that large values of $\Delta m^{2}$ are favored. This is confirmed by the values of the integral probabilities of large $\left(>10^{-5} \mathrm{eV}^{2}\right)$ and small $\left(<10^{-5} \mathrm{eV}^{2}\right)$ values of $\Delta m^{2}$ listed in Table III, that are comparable to those obtained in the model dependent analysis, also reported in Table III.

The model independent indications in favor of large mixing and large values of $\Delta m^{2}$ are very important for future terrestrial experiments that could explore this region of the parameter space with reactor neutrinos [78, 79].

\section{Neutrino Fluxes}

In this section we present the information on neutrino fluxes obtained through the marginal posterior distributions

$$
p\left(\Phi_{i} \mid \mathrm{D}, \mathrm{I}\right)=\int \prod_{i^{\prime} \neq i} \mathrm{~d} \Phi_{i^{\prime}} \int \mathrm{d} \mathbf{C} \int \mathrm{d} \log \left(\tan ^{2} \vartheta\right) \mathrm{d} \log \left(\Delta m^{2}\right) p\left(\tan ^{2} \vartheta, \Delta m^{2}, \Phi, \mathbf{C} \mid \mathrm{D}, \mathrm{I}\right) .
$$

As could have been expected, we found that only the posterior distributions of the $p p,{ }^{7} \mathrm{Be}$ and ${ }^{8} \mathrm{~B}$ fluxes, to which the existing experiments are most sensitive, are significantly different from the corresponding prior distributions. The posterior distributions of all the other fluxes, including hep, is practically flat in the same range as the prior distribution.

These distributions of the $p p,{ }^{7} \mathrm{Be}$ and ${ }^{8} \mathrm{~B}$ fluxes are shown in Figs. 15 20. In all these figures the thick solid line is the model independent posterior distribution, the thick dashed line is the prior distribution and the thick dotted line is the Gaussian BP2000 Standard Solar Model distribution. The intervals in which the thick solid line lies above the thin horizontal dotted, dashed and solid lines have, respectively, 90\%, 95\% and 99\% probability. The thick solid lines representing the posterior distributions in Figs. 1520 have small fluctuations because the integrals over the fluxes and cross sections in Eq. (34) have been performed through a Monte Carlo. The fluctuations of the thick dashed lines is due to the fact that the prior distribution is generated by the Monte Carlo.

Table VII gives the credible intervals of the $p p,{ }^{7} \mathrm{Be}$ and ${ }^{8} \mathrm{~B}$ fluxes with $90 \%, 95 \%$ and $99 \%$ probability in the Rates and Global Analyses.

Figures 15 and 16 show that the posterior distribution of the $p p$ flux is in good agreement with the SSM distribution, with a slight preference for high values. The upper limit of the credible intervals with $90 \%, 95 \%$ and $99 \%$ probability reported in Table VII does not give a significant information, since it almost coincides with the upper limit of the prior distribution, that is determined by the luminosity constraint (30), as explained in Section $\mathrm{VA}$. On the other hand, the lower limit of the credible intervals reported in Table VII is interesting, because it is significantly larger than the lower limit of the prior distribution, that follows from the prior distributions of the other fluxes and the luminosity constraint (30). In other words, the existing solar neutrino data allow to improve our knowledge of the $p p$ flux, increasing its allowed lower limit.

Also the posterior distributions of the ${ }^{7}$ Be flux shown in Figures 17 and 18 are in good agreement with the SSM distribution, with a slight preference for low values. One can see from Table VI that, according to the model independent analysis of solar neutrino data, the ${ }^{7} \mathrm{Be}$ flux could be very small, but it is limited from above. Especially the Global Analysis restricts the allowed upper value for the ${ }^{7} \mathrm{Be}$ flux significantly below the upper value of the prior distribution, as one can see from Fig. 18 and Table VII. Let us remember that the ${ }^{7}$ Be neutrino flux has also been constrained in Ref. 80 within $\pm 25 \%$ ( $1 \sigma$ error) of the SSM value from helioseismological measurements.

Finally, Figures 19 and 20 show that the posterior distribution of the ${ }^{8} \mathrm{~B}$ flux is in excellent agreement with the SSM distribution and it is bounded both from above and from below in an interval smaller than the prior distribution. This is a very interesting result, that agrees with the ranges for the ${ }^{8} \mathrm{~B}$ flux obtained in Refs. [2, 3, $\left.1,5,5,17,20,24,26,29\right]$ from the comparison of the SNO and Super-Kamiokande total rates only, and in Ref. [24, 29] from the least-squares analysis of all solar neutrino data with the ${ }^{8} \mathrm{~B}$ flux considered as a free parameter. For example, the authors of Ref. [3] found $0.44 \lesssim \Phi_{8_{\mathrm{B}}} / \Phi_{8_{\mathrm{B}}}^{\mathrm{SSM}} \lesssim 1.62$ at $3 \sigma$, i.e. $99.73 \%$ confidence level. We find that the credible interval of $\Phi_{8_{\mathrm{B}}} / \Phi_{8_{\mathrm{B}}}^{\mathrm{SSM}}$ with $99.73 \%$ probability is

$$
0.42<\Phi_{8_{\mathrm{B}}} / \Phi_{8_{\mathrm{B}}}^{\mathrm{SSM}}<1.75
$$

in the Rates Analysis and

$$
0.45<\Phi_{8_{\mathrm{B}}} / \Phi_{8_{\mathrm{B}}}^{\mathrm{SSM}}<1.42
$$

in the Global Analysis. Although the meaning of confidence level intervals and credible intervals is different, it is fair to say that the Global Analysis allows to restrict the allowed range of the ${ }^{8} \mathrm{~B}$ neutrino flux more than the comparison of the SNO and Super-Kamiokande total rates only. 


\section{Oscillations versus no oscillations}

Bayesian Probability Theory allows to compare the compatibility of different hypotheses with the observed data calculating the ratio of their probabilities, that is usually called "odds". Hence, we can compare in a model independent way the oscillation hypothesis with the no oscillation hypothesis, i.e. the Standard Model of neutrino physics.

In order to perform this task we must reconsider Bayes Theorem in the form (ब). The posterior probability of the oscillation hypothesis is given by

$$
\begin{aligned}
& p(\mathrm{OSC} \mid \mathrm{D}, \mathrm{I})=\int \mathrm{d} \log \left(\tan ^{2} \vartheta\right) \mathrm{d} \log \left(\Delta m^{2}\right) \int \mathrm{d} \Phi \int \mathrm{d} \mathbf{C} p\left(\tan ^{2} \vartheta, \Delta m^{2}, \Phi, \mathbf{C} \mid \mathrm{D}, \mathrm{I}\right) \\
& =\frac{\int \mathrm{d} \log \left(\tan ^{2} \vartheta\right) \mathrm{d} \log \left(\Delta m^{2}\right) \int \mathrm{d} \Phi \int \mathrm{d} \mathbf{C} p\left(\mathrm{D} \mid \tan ^{2} \vartheta, \Delta m^{2}, \Phi, \mathbf{C}, \mathrm{I}\right) p\left(\tan ^{2} \vartheta, \Delta m^{2} \mid \mathrm{I}\right) p(\Phi \mid \mathrm{I}) p(\mathbf{C} \mid \mathrm{I})}{p(\mathrm{D} \mid \mathrm{I})} .
\end{aligned}
$$

The flat prior distribution in the $\log \left(\tan ^{2} \vartheta\right)-\log \left(\Delta m^{2}\right)$ plane of Figs. 1 9 is given by

$$
p\left(\tan ^{2} \vartheta, \Delta m^{2} \mid \mathrm{I}\right)=\frac{1}{\Delta \log \left(\tan ^{2} \vartheta\right) \Delta \log \left(\Delta m^{2}\right)},
$$

where $\Delta \log \left(\tan ^{2} \vartheta\right)$ and $\Delta \log \left(\Delta m^{2}\right)$ are the logarithmic ranges of $\tan ^{2} \vartheta$ and $\Delta m^{2}: \Delta \log \left(\tan ^{2} \vartheta\right)=7$ and $\Delta \log \left(\Delta m^{2}\right)=$ 11.

We compare the probability of neutrino oscillations (37) with the probability of no oscillations

$$
p(\mathrm{NO}-\mathrm{OSC} \mid \mathrm{D}, \mathrm{I})=\frac{\int \mathrm{d} \Phi \int \mathrm{d} \mathbf{C} p\left(\mathrm{D} \mid \tan ^{2} \vartheta=0, \Delta m^{2}=0, \Phi, \mathbf{C}, \mathrm{I}\right) p(\Phi \mid \mathrm{I}) p(\mathbf{C} \mid \mathrm{I})}{p(\mathrm{D} \mid \mathrm{I})} .
$$

Notice that in the ratio of Eqs. (39) and (37) the unknown probability $p(\mathrm{D} \mid \mathrm{I})$ cancels.

Our result for the comparison of no neutrino oscillations versus neutrino oscillations is

$$
\frac{p(\mathrm{NO}-\mathrm{OSC} \mid \mathrm{D}, \mathrm{I})}{p(\mathrm{OSC} \mid \mathrm{D}, \mathrm{I})}=8.7 \times 10^{-4}
$$

in the Rates Analysis and

$$
\frac{p(\mathrm{NO}-\mathrm{OSC} \mid \mathrm{D}, \mathrm{I})}{p(\mathrm{OSC} \mid \mathrm{D}, \mathrm{I})}=7.4 \times 10^{-4} .
$$

in the Global Analysis. Therefore, there is a rather strong model independent indication in favor of neutrino oscillations, in agreement with those obtained in Refs. 2, 3, 4, 5, 6. The odds in favor of neutrino oscillations are slightly stronger in the Rates Analysis than in the Global Analysis because the Super-Kamiokande energy spectrum is rather flat, which would be in agreement with the hypothesis of no oscillations if the Super-Kamiokande rate were equal to the SNO rate.

Finally, let us notice that the prior distribution (38) and the result of the comparison of the oscillation and nooscillation hypotheses that we have calculated depends on the ranges $\Delta \log \left(\tan ^{2} \vartheta\right)$ and $\Delta \log \left(\Delta m^{2}\right)$. However, one must always keep in mind that these ranges have not been chosen randomly, but follow from our prior knowledge that there can be a measurable oscillation effect in solar neutrino experiments only if the neutrino mixing parameters lie within them. It is possible that somebody may argue in favor of a slight expansion or contraction of the prior ranges of $\Delta \log \left(\tan ^{2} \vartheta\right)$ and $\Delta \log \left(\Delta m^{2}\right)$ that we have considered. However, the effect of such expansion or contraction on the result of the comparison of the oscillation and no-oscillation hypotheses is small, not changing its order of magnitude.

\section{CONCLUSIONS}

In this paper we have analyzed the data of solar neutrino experiment in terms of $\nu_{e} \rightarrow \nu_{\mu, \tau}$ oscillations without assuming the Standard Solar Model values of the neutrino fluxes. Working in the framework of Bayesian Probability Theory, the assumed lack of knowledge of the neutrino fluxes has been quantified through a flat prior probability distribution for the neutrino fluxes, that have been considered as unknown quantities to be determined, if possible, from the analysis of the data.

In Section IV] we have checked the reliability of our approach using a multi-normal prior probability distribution for the neutrino fluxes corresponding to the prediction of the BP2000 Standard Solar Model. We have shown that the 
resulting allowed regions for the neutrino mixing parameters $\tan ^{2} \vartheta, \Delta m^{2}$ are practically equal with those obtained with the standard method in which the uncertainties of the fluxes are taken into account in the covariance matrix that determines the likelihood function.

The results of the model independent analysis presented in Section $\square$ show that the present solar neutrino data allow to derive rather stringent model independent information on the neutrino mixing parameters, improving dramatically the conclusions obtained several years ago [42] in another model independent analysis of solar neutrino data.

Figures 3 and 9, obtained with the Rates Analysis and Global Analysis, respectively, show that the allowed regions of the neutrino mixing parameters are rather restricted and not much larger than those obtained in the SSM model dependent analysis (shown in Figs. 1 and 2). One can distinguish clearly the usual SMA, LMA, LOW and VO regions. Therefore, we conclude that the indication that the true values of the neutrino mixing parameters lie in one of these regions is robust.

We have also shown in Section VB that there is a strong model independent indication in favor of large neutrino mixing and large values of $\Delta m^{2}$. This is very important for future terrestrial experiments that could explore this part of the parameter space of neutrino mixing [78, 79. In particular, the model independent Global Analysis strongly disfavors the SMA solution of the solar neutrino problem. This is an impressive consequence of the high quality of the existing data.

In Section $\mathrm{VC}$ we have presented the posterior distributions of the $p p,{ }^{7} \mathrm{Be}$ and ${ }^{8} \mathrm{~B}$ neutrino fluxes, to which the existing data are most sensitive (the posterior distribution of the other fluxes is similar to the prior distribution). We have shown that the posterior distributions of these fluxes are in excellent agreement with the BP2000 Standard Solar Model distribution, with a slight preference of a high $p p$ flux and a low ${ }^{7} \mathrm{Be}$ flux. The ${ }^{8} \mathrm{~B}$ is severely constrained in an interval around the SSM value (see Table VII and Eqs. (35), (36)), in agreement with the limits found in Refs. [2, 3, 4, 5, 24, 26, 29].

Finally, in Section $\mathrm{VD}$ we have shown that the hypothesis of no neutrino oscillations is strongly disfavored with respect to the hypothesis of $\nu_{e} \rightarrow \nu_{\mu, \tau}$ oscillations, in agreement with the results obtained in Refs. [2, 3, 4, 5, 6].

In conclusion, we would like to emphasize the usefulness of the quantity and quality of the existing solar neutrino data, that nowadays allow to obtain stringent model independent information on neutrino physics and solar physics. We enthusiastically look forward towards the realization of new more sensitive solar neutrino experiments in order to improve our knowledge.

\section{Acknowledgments}

We would like to express our gratitude to M.C. Gonzalez-Garcia and C. Pena-Garay for useful discussions on the analysis of solar neutrino data. M.V. Garzelli thanks A. Palazzo for very useful discussions and suggestions and A. Bottino for encouragments and support. C. Giunti would like to thank the Korea Institute for Advanced Study (KIAS) for warm hospitality during the final part of this work.

[1] S. Fukuda et al. (SuperKamiokande), Phys. Rev. Lett. 86, 5651 (2001), hep-ex/0103032.

[2] Q. R. Ahmad et al. (SNO), Phys. Rev. Lett. 87, 071301 (2001), nucl-ex/0106015.

[3] G. L. Fogli, E. Lisi, D. Montanino, and A. Palazzo, Phys. Rev. D64, 093007 (2001), hep-ph/0106247.

[4] C. Giunti (2001), hep-ph/0107310.

[5] G. Fiorentini, F. L. Villante, and B. Ricci (2001), hep-ph/0109275.

[6] J. N. Bahcall (2001), hep-ph/0108147.

[7] B. T. Cleveland et al., Astrophys. J. 496, 505 (1998).

[8] W. Hampel et al. (GALLEX), Phys. Lett. B447, 127 (1999).

[9] V. N. Gavrin (SAGE), Nucl. Phys. Proc. Suppl. 91, 36 (2001).

[10] M. Altmann et al. (GNO), Phys. Lett. B490, 16 (2000), hep-ex/0006034.

[11] J. N. Bahcall, M. H. Pinsonneault, and S. Basu, Astrophys. J. 555, 990 (2001), astro-ph/0010346.

[12] S. M. Bilenky and B. Pontecorvo, Phys. Rept. 41, 225 (1978).

[13] S. M. Bilenky and S. T. Petcov, Rev. Mod. Phys. 59, 671 (1987).

[14] C. W. Kim and A. Pevsner, Neutrinos in physics and astrophysics (Harwood Academic Press, Chur, Switzerland, 1993), Contemporary Concepts in Physics, Vol. 8.

[15] S. M. Bilenky, C. Giunti, and W. Grimus, Prog. Part. Nucl. Phys. 43, 1 (1999), hep-ph/9812360.

[16] V. Barger, D. Marfatia, and K. Whisnant (2001), hep-ph/0106207.

[17] J. N. Bahcall, M. C. Gonzalez-Garcia, and C. Pena-Garay, JHEP 08, 014 (2001), hep-ph/0106258.

[18] A. Bandyopadhyay, S. Choubey, S. Goswami, and K. Kar (2001), hep-ph/0106264.

[19] P. Creminelli, G. Signorelli, and A. Strumia (2001), hep-ph/0102234. 
[20] R. Barbieri and A. Strumia (2000), hep-ph/0011307.

[21] M. Kachelriess, A. Strumia, R. Tomas, and J. W. F. Valle (2001), hep-ph/0108100.

[22] V. Berezinsky and M. Lissia (2001), hep-ph/0108108.

[23] V. Berezinsky (2001), hep-ph/0108166.

[24] P. I. Krastev and A. Y. Smirnov (2001), hep-ph/0108177.

[25] M. V. Garzelli and C. Giunti (2001), hep-ph/0108191.

[26] M. B. Smy (2001), arXiv:hep-ex/0108053.

[27] S. M. Bilenky, T. Lachenmaier, W. Potzel, and F. von Feilitzsch (2001), hep-ph/0109200.

[28] A. Strumia and F. Vissani (2001), hep-ph/0109172.

[29] J. N. Bahcall, M. C. Gonzalez-Garcia, and C. Pena-Garay (2001), hep-ph/0111150.

[30] J. N. Bahcall, Neutrino Astrophysics (Cambridge University Press, Cambridge, UK, 1989).

[31] S. M. Bilenky and C. Giunti, Phys. Lett. B444, 379 (1998), hep-ph/9802201.

[32] G. L. Fogli, E. Lisi, D. Montanino, and A. Palazzo, Phys. Rev. D62, 013002 (2000), hep-ph/9912231.

[33] M. Apollonio et al. (CHOOZ), Phys. Lett. B466, 415 (1999), hep-ex/9907037.

[34] V. Barger, B. Kayser, J. Learned, T. Weiler, and K. Whisnant, Phys. Lett. B489, 345 (2000), hep-ph/0008019.

[35] C. Giunti and M. Laveder, JHEP 02, 001 (2001), hep-ph/0010009.

[36] C. M. Bhat, P. C. Bhat, M. Paterno, and H. B. Prosper, Phys. Rev. Lett. 81, 5056 (1998), astro-ph/9804252.

[37] H. Jeffreys, Theory of Probability (Oxford University Press, New York, USA, 1961), first published in 1939.

[38] T. J. Loredo (1990), in Maximum-Entropy and Bayesian Methods, Dartmouth, 1989, ed. P. Fougere, Kluwer Academic Publishers, Dordrecht, The Netherlands, 1990, pp. 81-142, http://astrosun.tn.cornell.edu/staff/loredo/bayes/tjl.html.

[39] T. J. Loredo (1992), in Statistical Challenges in Modern Astronomy, ed. E.D. Feigelson and G.J. Babu, Springer-Verlag, New York, 1992, pp. 275-297, http://astrosun.tn.cornell.edu/staff/loredo/bayes/tjl.html.

[40] E. T. Jaynes, Probability Theory: The Logic of Science (Fragmentary Edition, June 1994), http://bayes.wustl.edu/etj/prob.html.

[41] G. D'Agostini, CERN Yellow Report 99-03 (1999).

[42] S. M. Bilenky and C. Giunti (1994), hep-ph/9407379.

[43] V. N. Gribov and B. Pontecorvo, Phys. Lett. B28, 493 (1969).

[44] L. Wolfenstein, Phys. Rev. D17, 2369 (1978).

[45] S. P. Mikheev and A. Y. Smirnov, Sov. J. Nucl. Phys. 42, 913 (1985).

[46] P. Creminelli, G. Signorelli, and A. Strumia, JHEP 05, 052 (2001), hep-ph/0102234.

[47] M. Apollonio et al. (CHOOZ), Phys. Lett. B420, 397 (1998), hep-ex/9711002.

[48] J. Bahcall (2001), WWW page: http://www.sns.ias.edu/ jnb.

[49] S. Nakamura, T. Sato, V. Gudkov, and K. Kubodera, Phys. Rev. C63, 034617 (2001), nucl-th/0009012.

[50] K. Kubodera (2001), WWW page: http://nuc003.psc.sc.edu/ kubodera/NU-D-NSGK.

[51] M. Butler, J.-W. Chen, and X. Kong, Phys. Rev. C63, 035501 (2001), nucl-th/0008032.

[52] J. F. Beacom and S. J. Parke, Phys. Rev. D64, 091302 (2001), hep-ph/0106128.

[53] A. Kurylov, M. J. Ramsey-Musolf, and P. Vogel (2001), nucl-th/0110051.

[54] A. W. P. Poon (SNO) (2001), nucl-ex/0110005.

[55] A. Friedland, Phys. Rev. Lett. 85, 936 (2000), hep-ph/0002063.

[56] G. L. Fogli, E. Lisi, D. Montanino, and A. Palazzo, Phys. Rev. D62, 113004 (2000), hep-ph/0005261.

[57] E. Lisi, A. Marrone, D. Montanino, A. Palazzo, and S. T. Petcov, Phys. Rev. D63, 093002 (2001), hep-ph/0011306.

[58] S. T. Petcov, Phys. Lett. B214, 139 (1988).

[59] S. T. Petcov and J. Rich, Phys. Lett. B224, 426 (1989).

[60] M. C. Gonzalez-Garcia and C. Pena-Garay, Nucl. Phys. Proc. Suppl. 91, 80 (2000), hep-ph/0009041.

[61] S. T. Petcov, Phys. Lett. B200, 373 (1988).

[62] T. K. Kuo and J. Pantaleone, Rev. Mod. Phys. 61, 937 (1989).

[63] Q. Y. Liu, M. Maris, and S. T. Petcov, Phys. Rev. D56, 5991 (1997), hep-ph/9702361.

[64] S. T. Petcov, Phys. Lett. B434, 321 (1998), hep-ph/9805262.

[65] E. K. Akhmedov, Nucl. Phys. B538, 25 (1999), hep-ph/9805272.

[66] M. V. Chizhov and S. T. Petcov, Phys. Rev. Lett. 83, 1096 (1999), hep-ph/9903399.

[67] M. V. Chizhov and S. T. Petcov, Phys. Rev. D63, 073003 (2001), hep-ph/9903424.

[68] S. Fukuda et al. (SuperKamiokande) (2001), hep-ex/0103032.

[69] C. E. Ortiz, A. Garcia, R. A. Waltz, M. Bhattacharya, and A. K. Komives, Phys. Rev. Lett. 85, 2909 (2000), nuclex/0003006.

[70] J. N. Bahcall et al., Phys. Rev. C54, 411 (1996), nucl-th/9601044.

[71] M. V. Garzelli and C. Giunti, Phys. Lett. B488, 339 (2000), hep-ph/0006026.

[72] G. L. Fogli and E. Lisi, Astropart. Phys. 3, 185 (1995).

[73] J. N. Bahcall, Nucl. Phys. Proc. Suppl. 91, 9 (2000), hep-ph/0009044.

[74] J. N. Bahcall and P. I. Krastev, Phys. Lett. B436, 243 (1998), hep-ph/9807525.

[75] J. N. Bahcall (2001), hep-ph/0108148.

[76] J. N. Bahcall, P. I. Krastev, and A. Y. Smirnov, JHEP 05, 015 (2001), hep-ph/0103179.

[77] A. de Gouvea, A. Friedland, and H. Murayama, Phys. Lett. B490, 125 (2000), hep-ph/0002064.

[78] A. Piepke (KamLAND), Nucl. Phys. Proc. Suppl. 91, 99 (2001).

[79] G. Ranucci et al. (BOREXINO), Nucl. Phys. Proc. Suppl. 91, 58 (2001). 
[80] B. Ricci and F. L. Villante, Phys. Lett. B488, 123 (2000), astro-ph/0005538.

[81] J. N. Bahcall, S. Basu, and M. H. Pinsonneault, Phys. Lett. B433, 1 (1998), astro-ph/9805135.

[82] J. N. Bahcall and M. H. Pinsonneault, Rev. Mod. Phys. 67, 781 (1995), hep-ph/9505425.

[83] S. T. Chieze and I. Lopes, Astrophys. J. 408, 347 (1993).

[84] S. T. Chieze, W. Dappen, E. Fossat, J. Provostand, E. Schatzman, and D. Vignaud, Phys. Rept. 230, 57 (1993).

[85] J. N. Bahcall and M. H. Pinsonneault, Rev. Mod. Phys. 64, 885 (1992).

[86] J. N. Bahcall and R. K. Ulrich, Rev. Mod. Phys. 60, 297 (1988). 


\begin{tabular}{|c|c|c|}
\hline$j$ & Detection Material and Process & Data \\
\hline 1 & $\begin{array}{ll}{ }^{37} \mathrm{Cl}: & \nu_{e}+{ }^{37} \mathrm{Cl} \rightarrow{ }^{37} \mathrm{Ar}+e^{-} \\
& (\text {Homestake 胞) }\end{array}$ & $2.56 \pm 0.23 \mathrm{SNU}$ \\
\hline 2 & $\begin{array}{c}{ }^{71} \mathrm{Ga}: \quad \nu_{e}+{ }^{71} \mathrm{Ga} \rightarrow{ }^{71} \mathrm{Ge}+e^{-} \\
\text {(GALLEX }[\mathbb{\beta}]+\text { GNO }[10]+\text { SAGE }[9])\end{array}$ & $74.7 \pm 5.1 \mathrm{SNU}$ \\
\hline 3 & $\begin{array}{cc}\mathrm{D}_{2} \mathrm{O}: & \nu_{e}+d \vec{p} p+p+e^{-} \\
& (\mathrm{SNO} \text { [2]) }\end{array}$ & $0.347 \pm 0.028$ \\
\hline 4 & $\begin{array}{l}\mathrm{H}_{2} \mathrm{O}: \quad \nu+e^{-} \rightarrow \nu+e^{-} \\
\text {(Super-Kamiokande [1]) }\end{array}$ & $0.459 \pm 0.017$ \\
\hline
\end{tabular}

TABLE I: The rates measured in solar neutrino experiments. The rates of the Homestake and GALLEX+SAGE+GNO experiments are expressed in SNU units $\left(1 \mathrm{SNU} \equiv 10^{-36}\right.$ events atom $\left.{ }^{-1} \mathrm{~s}^{-1}\right)$, whereas the results of the Kamiokande and SNO experiments are expressed in terms of the ratio of the experimental rate and the BP2000 Standard Solar Model prediction [11. The statistical and systematic uncertainties have been added in quadrature. The GALLEX+SAGE+GNO rate is a weighted average of the GALLEX+GNO rate reported in Ref. [10] and the SAGE rate reported in Ref. [9]. The rate of the SNO experiment is that measured through $\mathrm{CC}$ weak interactions.

\begin{tabular}{|c|c|c|c|c|c|}
\hline $\begin{array}{c}b \\
\text { day }\end{array}$ & $\begin{array}{c}b \\
\text { day }\end{array}$ & $\begin{array}{c}K_{3 b}^{0} \\
\left(\mathrm{~cm}^{2} \mathrm{~s}\right)\end{array}$ & $\begin{array}{c}K_{5 b}^{0} \\
\left(\mathrm{~cm}^{2} \mathrm{~s}\right)\end{array}$ & $\delta_{b, \text { cor }}^{\text {(th) }}$ & $\delta_{b, \text { cor }}^{\text {(sys) }}$ \\
\hline 1 & 20 & $6.21 \times 10^{-8}$ & $1.98 \times 10^{-7}$ & 0.0039 & 0.0020 \\
\hline 2 & 21 & $6.62 \times 10^{-8}$ & $1.98 \times 10^{-7}$ & 0.0039 & 0.0020 \\
\hline 3 & 22 & $7.09 \times 10^{-8}$ & $1.98 \times 10^{-7}$ & 0.0047 & 0.0029 \\
\hline 4 & 23 & $7.63 \times 10^{-8}$ & $1.98 \times 10^{-7}$ & 0.0055 & 0.0058 \\
\hline 5 & 24 & $8.22 \times 10^{-8}$ & $1.98 \times 10^{-7}$ & 0.0062 & 0.0077 \\
\hline 6 & 25 & $9.23 \times 10^{-8}$ & $1.98 \times 10^{-7}$ & 0.0070 & 0.0106 \\
\hline 7 & 26 & $1.00 \times 10^{-7}$ & $1.98 \times 10^{-7}$ & 0.0078 & 0.0134 \\
\hline 8 & 27 & $1.12 \times 10^{-7}$ & $1.98 \times 10^{-7}$ & 0.0086 & 0.0163 \\
\hline 9 & 28 & $1.29 \times 10^{-7}$ & $1.98 \times 10^{-7}$ & 0.0093 & 0.0203 \\
\hline 10 & 29 & $1.47 \times 10^{-7}$ & $1.98 \times 10^{-7}$ & 0.0101 & 0.0242 \\
\hline 11 & 30 & $1.72 \times 10^{-7}$ & $1.98 \times 10^{-7}$ & 0.0109 & 0.0292 \\
\hline 12 & 31 & $2.03 \times 10^{-7}$ & $1.98 \times 10^{-7}$ & 0.0117 & 0.0331 \\
\hline 13 & 32 & $2.44 \times 10^{-7}$ & $1.98 \times 10^{-7}$ & 0.0124 & 0.0381 \\
\hline 14 & 33 & $2.91 \times 10^{-7}$ & $1.97 \times 10^{-7}$ & 0.0132 & 0.0440 \\
\hline 15 & 34 & $3.72 \times 10^{-7}$ & $1.97 \times 10^{-7}$ & 0.0140 & 0.0500 \\
\hline 16 & 35 & $4.72 \times 10^{-7}$ & $1.97 \times 10^{-7}$ & 0.0148 & 0.0570 \\
\hline 17 & 36 & $6.08 \times 10^{-7}$ & $1.97 \times 10^{-7}$ & 0.0155 & 0.0640 \\
\hline 18 & 37 & $8.21 \times 10^{-7}$ & $1.97 \times 10^{-7}$ & 0.0163 & 0.0730 \\
\hline 19 & 38 & $1.95 \times 10^{-6}$ & $1.94 \times 10^{-7}$ & 0.0205 & 0.1058 \\
\hline
\end{tabular}

TABLE II: Values of the relative theoretical uncertainties $\delta_{b, \text { cor }}^{(\text {th })}$ and experimental uncertainties $\delta_{b, \text { cor }}^{\text {(sys) }}$ of the Super-Kamiokande energy bins used in the Global Analysis (see Section IIB]). 


\begin{tabular}{|c|c|c|c|c|}
\hline \multirow{2}{*}{} & \multicolumn{2}{|c|}{ BP2000 SSM } & \multicolumn{2}{c|}{ Model Independent } \\
\cline { 2 - 5 } & Rates & Global & Rates & Global \\
\hline SMA & $9.9 \times 10^{-2}$ & $7.2 \times 10^{-5}$ & 0.15 & $1.0 \times 10^{-3}$ \\
\hline LMA & 0.72 & 0.86 & 0.67 & 0.80 \\
\hline LOW & $6.1 \times 10^{-2}$ & 0.12 & $3.8 \times 10^{-2}$ & 0.13 \\
\hline VO & 0.12 & $1.9 \times 10^{-2}$ & 0.14 & $6.6 \times 10^{-2}$ \\
\hline $\tan ^{2} \vartheta<0.1$ & $9.9 \times 10^{-2}$ & $7.2 \times 10^{-5}$ & 0.18 & $8.2 \times 10^{-3}$ \\
\hline $0.1<\tan ^{2} \vartheta<10$ & 0.90 & 1.00 & 0.82 & 0.99 \\
\hline $\tan ^{2} \vartheta>10$ & $6.5 \times 10^{-8}$ & $1.3 \times 10^{-8}$ & $1.1 \times 10^{-3}$ & $1.7 \times 10^{-3}$ \\
\hline$\Delta m^{2}<10^{-5} \mathrm{eV}^{2}$ & 0.32 & 0.14 & 0.41 & 0.20 \\
\hline$\Delta m^{2}>10^{-5} \mathrm{eV}^{2}$ & 0.68 & 0.86 & 0.59 & 0.80 \\
\hline
\end{tabular}

TABLE III: Integral probabilities of regions in the $\tan ^{2} \vartheta-\Delta m^{2}$ plane and of intervals of $\tan ^{2} \vartheta$ and $\Delta m^{2}$ obtained with the model dependent and model independent Rates Analysis and Global Analysis. The SMA, LMA, LOW and VO regions are defined in Eqs. (7)-(10).

\begin{tabular}{|c|c|c|c|c|c|c|c|c|}
\hline$i$ & $\begin{array}{c}1 \\
p p\end{array}$ & $\begin{array}{c}2 \\
p e p\end{array}$ & $\begin{array}{c}3 \\
h e p\end{array}$ & $\begin{array}{c}4 \\
{ }^{7} \mathrm{Be}\end{array}$ & $\begin{array}{c}5 \\
{ }^{8} \mathrm{~B}\end{array}$ & $\begin{array}{c}6 \\
13 \mathrm{~N}\end{array}$ & $\begin{array}{c}7 \\
15 \mathrm{O}\end{array}$ & $\begin{array}{c}8 \\
17 \mathrm{~F}\end{array}$ \\
\hline$\Delta \Phi_{i} / \Phi_{i}^{\mathrm{SSM}}$ & $\begin{array}{l}+0.094 \\
-0.096\end{array}$ & \pm 1 & $\begin{array}{l}+9 \\
-1\end{array}$ & \pm 1 & \pm 1 & \pm 1 & \pm 1 & \pm 1 \\
\hline$\left(\Delta \Phi_{i}^{\mathrm{SSM}} / \Phi_{i}^{\mathrm{SSM}}\right)_{(1 \sigma)}$ & \pm 0.010 & \pm 0.015 & - & \pm 0.100 & \pm 0.200 & \pm 0.210 & \pm 0.250 & \pm 0.250 \\
\hline $\operatorname{NACRE}(3.842)$ [1] & 0.002 & -0.007 & 0.011 & 0.008 & 0.077 & -0.111 & -0.129 & -0.059 \\
\hline BP2000 (3.844) & 0.002 & 0.000 & 0.000 & 0.010 & 0.020 & 0.015 & 0.017 & 0.018 \\
\hline $\operatorname{NACRE}(3.844)$ 11] & 0.003 & -0.007 & 0.011 & 0.017 & 0.097 & -0.100 & -0.117 & -0.043 \\
\hline AS00 [11] & 0.007 & 0.007 & 0.011 & -0.031 & -0.069 & -0.042 & -0.050 & -0.053 \\
\hline GN 93 11 & -0.002 & -0.007 & -0.011 & 0.023 & 0.051 & 0.128 & 0.135 & 0.155 \\
\hline Pre-M.S. 11 & 0.000 & -0.007 & -0.011 & 0.021 & 0.048 & 0.124 & 0.131 & 0.149 \\
\hline Rotation 11$]$ & 0.005 & 0.000 & -0.011 & -0.019 & -0.028 & 0.016 & 0.015 & 0.028 \\
\hline Radius78 [11] & -0.002 & -0.007 & -0.011 & 0.023 & 0.051 & 0.128 & 0.135 & 0.155 \\
\hline \begin{tabular}{l|l} 
Radius$_{508}$ & 11 \\
\end{tabular} & -0.002 & -0.007 & -0.011 & 0.023 & 0.051 & 0.128 & 0.135 & 0.155 \\
\hline No Diffusion [1] & 0.017 & 0.021 & 0.032 & -0.117 & -0.234 & -0.254 & -0.279 & -0.281 \\
\hline Old Physics 11$]$ & 0.000 & 007 & 0.011 & 029 & 0.020 & 0.053 & 0.048 & 0.052 \\
\hline$S_{3,4}=011$ & 0.076 & 0.107 & 0.086 & -1.000 & -1.000 & 0.181 & 0.175 & 0.190 \\
\hline Mixed 11 & 0.030 & -0.093 & -0.333 & -0.252 & -0.182 & -0.445 & -0.365 & -0.359 \\
\hline BP98 81 & -0.002 & -0.007 & -0.774 & 0.006 & 0.020 & 0.104 & 0.108 & 0.124 \\
\hline BP95 82 & -0.007 & 0.000 & -0.871 & 0.080 & 0.311 & 0.128 & 0.135 & 0.151 \\
\hline DS94 & 0.017 & 0.039 & -0.866 & -0.107 & -0.298 & -0.074 & -0.083 & -0.211 \\
\hline TL93 83, 84 & 0.013 & -0.007 & - & -0.090 & -0.123 & -0.301 & -0.337 & - \\
\hline BP92 85 & 0.008 & 0.021 & -0.868 & 0.025 & 0.127 & -0.102 & -0.113 & -0.043 \\
\hline BU88 86 & 0.008 & 0.000 & -0.185 & -0.017 & 0.141 & 0.111 & 0.088 & -0.083 \\
\hline
\end{tabular}

TABLE IV: Relative uncertainties $\Delta \Phi_{i} / \Phi_{i}^{\mathrm{SSM}}$ of the prior distributions of the neutrino fluxes in the model independent analysis confronted with the relative uncertainties $\left(\Delta \Phi_{i}^{\mathrm{SSM}} / \Phi_{i}^{\mathrm{SSM}}\right)_{(1 \sigma)}$ in the BP2000 Standard Solar Model and the differences between the fluxes predicted by several models and the BP2000 SSM. 
Rates Analysis

\begin{tabular}{|c|c|c|c|c|c|c|c|c|c|}
\hline $\tan ^{2} \vartheta$ & $\Delta m^{2}\left(\mathrm{eV}^{2}\right)$ & $p p$ & $p e p$ & $h e p$ & ${ }^{7} \mathrm{Be}$ & ${ }^{8} \mathrm{~B}$ & ${ }^{13} \mathrm{~N}$ & ${ }^{15} \mathrm{O}$ & ${ }^{17} \mathrm{~F}$ \\
\hline $1.7 \times 10^{-3}$ & $8.0 \times 10^{-6}$ & 0.935 & 0.220 & 8.907 & 1.992 & 1.290 & 1.090 & 0.177 & 0.063 \\
\hline 0.20 & $1.2 \times 10^{-5}$ & 0.962 & 0.960 & 6.752 & 1.508 & 1.354 & 1.362 & 0.764 & 1.253 \\
\hline 0.49 & $1.4 \times 10^{-7}$ & 0.970 & 0.813 & 1.012 & 1.437 & 1.003 & 0.805 & 0.749 & 1.250 \\
\hline 3.59 & $8.0 \times 10^{-11}$ & 1.031 & 1.779 & 1.449 & 0.739 & 0.904 & 0.800 & 0.033 & 1.032 \\
\hline $1.0 \times 10^{-2}$ & $3.0 \times 10^{-5}$ & 1.035 & 1.344 & 5.116 & 0.349 & 1.981 & 1.967 & 1.861 & 1.632 \\
\hline $5.0 \times 10^{-2}$ & $1.0 \times 10^{-6}$ & 0.916 & 1.885 & 5.203 & 1.996 & 1.997 & 1.758 & 1.173 & 1.844 \\
\hline 1.00 & $9.0 \times 10^{-11}$ & 0.965 & 0.841 & 3.686 & 1.464 & 1.563 & 0.454 & 1.015 & 0.202 \\
\hline 1.00 & $5.5 \times 10^{-12}$ & 0.983 & 0.757 & 0.040 & 1.212 & 0.446 & 1.822 & 0.853 & 0.025 \\
\hline 0 & 0 & 1.089 & 0.679 & 0.249 & 0.021 & 0.428 & 0.233 & 0.103 & 0.481 \\
\hline
\end{tabular}

TABLE V: Values of the neutrino fluxes normalized to the BP2000 SSM fluxes corresponding to the lines in Fig. 8, obtained in the Rates Analysis with the nine selected values of $\tan ^{2} \vartheta$ and $\Delta m^{2}$ listed in the first two columns. The first four rows correspond, respectively, to the best-fit points in the SMA, LMA, LOW and VO regions in the Rates Analysis. The fifth to eighth rows correspond to points in excluded areas in Fig. 3. The last row corresponds to the case of no oscillations.

Global Analysis

\begin{tabular}{|c|c|c|c|c|c|c|c|c|c|}
\hline $\tan ^{2} \vartheta$ & $\Delta m^{2}\left(\mathrm{eV}^{2}\right)$ & $p p$ & $p e p$ & $h e p$ & ${ }^{7} \mathrm{Be}$ & ${ }^{8} \mathrm{~B}$ & ${ }^{13} \mathrm{~N}$ & ${ }^{15} \mathrm{O}$ & ${ }^{17} \mathrm{~F}$ \\
\hline $1.7 \times 10^{-3}$ & $8.0 \times 10^{-6}$ & 0.929 & 0.165 & 3.922 & 1.862 & 1.260 & 0.262 & 1.523 & 1.557 \\
\hline 0.20 & $1.2 \times 10^{-5}$ & 0.966 & 1.414 & 0.335 & 1.507 & 1.286 & 1.906 & 0.301 & 0.699 \\
\hline 0.49 & $1.4 \times 10^{-7}$ & 0.968 & 0.045 & 1.561 & 1.520 & 0.992 & 0.617 & 0.503 & 1.147 \\
\hline 3.59 & $8.0 \times 10^{-11}$ & 1.014 & 0.987 & 1.004 & 0.896 & 0.931 & 0.048 & 0.651 & 0.426 \\
\hline $3.8 \times 10^{-4}$ & $5.3 \times 10^{-6}$ & 1.035 & 0.697 & 2.764 & 0.535 & 0.526 & 0.394 & 1.186 & 0.114 \\
\hline 0.34 & $6.1 \times 10^{-5}$ & 1.004 & 1.248 & 5.544 & 1.047 & 1.104 & 1.854 & 0.186 & 0.433 \\
\hline 0.60 & $1.5 \times 10^{-7}$ & 0.994 & 0.147 & 5.910 & 1.223 & 0.915 & 0.319 & 0.342 & 0.967 \\
\hline 3.16 & $5.0 \times 10^{-10}$ & 1.050 & 1.111 & 4.100 & 0.510 & 0.620 & 0.008 & 0.141 & 0.255 \\
\hline 0 & 0 & 1.088 & 0.065 & 0.664 & 0.071 & 0.438 & 0.032 & 0.031 & 0.340 \\
\hline
\end{tabular}

TABLE VI: Values of the neutrino fluxes normalized to the BP2000 SSM fluxes corresponding to the lines in Fig. 10, obtained in the Global Analysis with the nine selected values of $\tan ^{2} \vartheta$ and $\Delta m^{2}$ listed in the first two columns. The first four rows correspond, respectively, to the best-fit points in the SMA, LMA, LOW and VO regions in the Rates Analysis. The fifth to eighth rows correspond, respectively, to the best-fit points in the SMA, LMA, LOW and VO regions in the Global Analysis. The last row corresponds to the case of no oscillations. 


\begin{tabular}{|c|c|c|c|c|c|c|}
\hline \multirow{2}{*}{} & \multicolumn{3}{|c|}{ Rates Analysis } & \multicolumn{3}{c|}{ Global Analysis } \\
\cline { 2 - 7 } & $90 \%$ & $95 \%$ & $99 \%$ & $90 \%$ & $95 \%$ & $99 \%$ \\
\hline$\Phi_{p p} / \Phi_{p p}^{\mathrm{SSM}}$ & {$[0.95,1.08]$} & {$[0.93,1.08]$} & {$[0.92,1.09]$} & {$[0.99,1.08]$} & {$[0.98,1.08]$} & {$[0.95,1.09]$} \\
\hline$\Phi_{7_{\mathrm{Be}}} / \Phi_{7 \mathrm{Be}}^{\mathrm{SSM}}$ & {$[0.02,1.65]$} & {$[0.02,1.82]$} & {$[0.02,1.95]$} & {$[0.02,1.15]$} & {$[0.02,1.28]$} & {$[0.02,1.62]$} \\
\hline$\Phi_{8_{\mathrm{B}}} / \Phi_{8_{\mathrm{B}}}^{\mathrm{SSM}}$ & {$[0.62,1.48]$} & {$[0.58,1.58]$} & {$[0.48,1.68]$} & {$[0.62,1.22]$} & {$[0.58,1.28]$} & {$[0.48,1.35]$} \\
\hline
\end{tabular}

TABLE VII: Credible intervals for the $p p,{ }^{7}$ Be and ${ }^{8} \mathrm{~B}$ fluxes with $90 \%, 95 \%$ and $99 \%$ probability obtained with the model independent Rates and Global Analyses. 


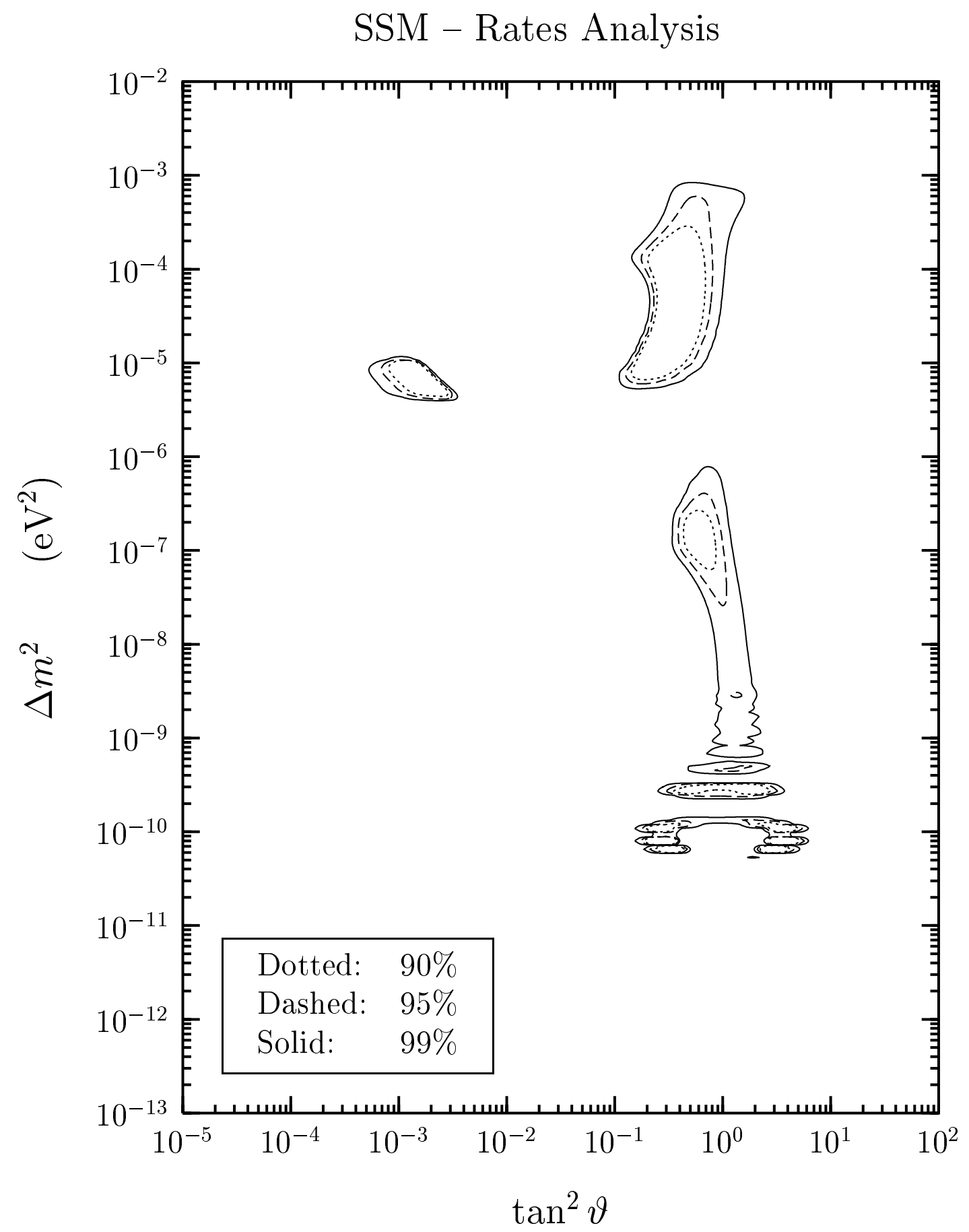

FIG. 1: Credible regions obtained with the Rates Analysis of Homestake, GALLEX+GNO+SAGE, SNO and SuperKamiokande total rates, assuming the BP2000 Standard Solar Model neutrino fluxes. 
SSM - Global Analysis

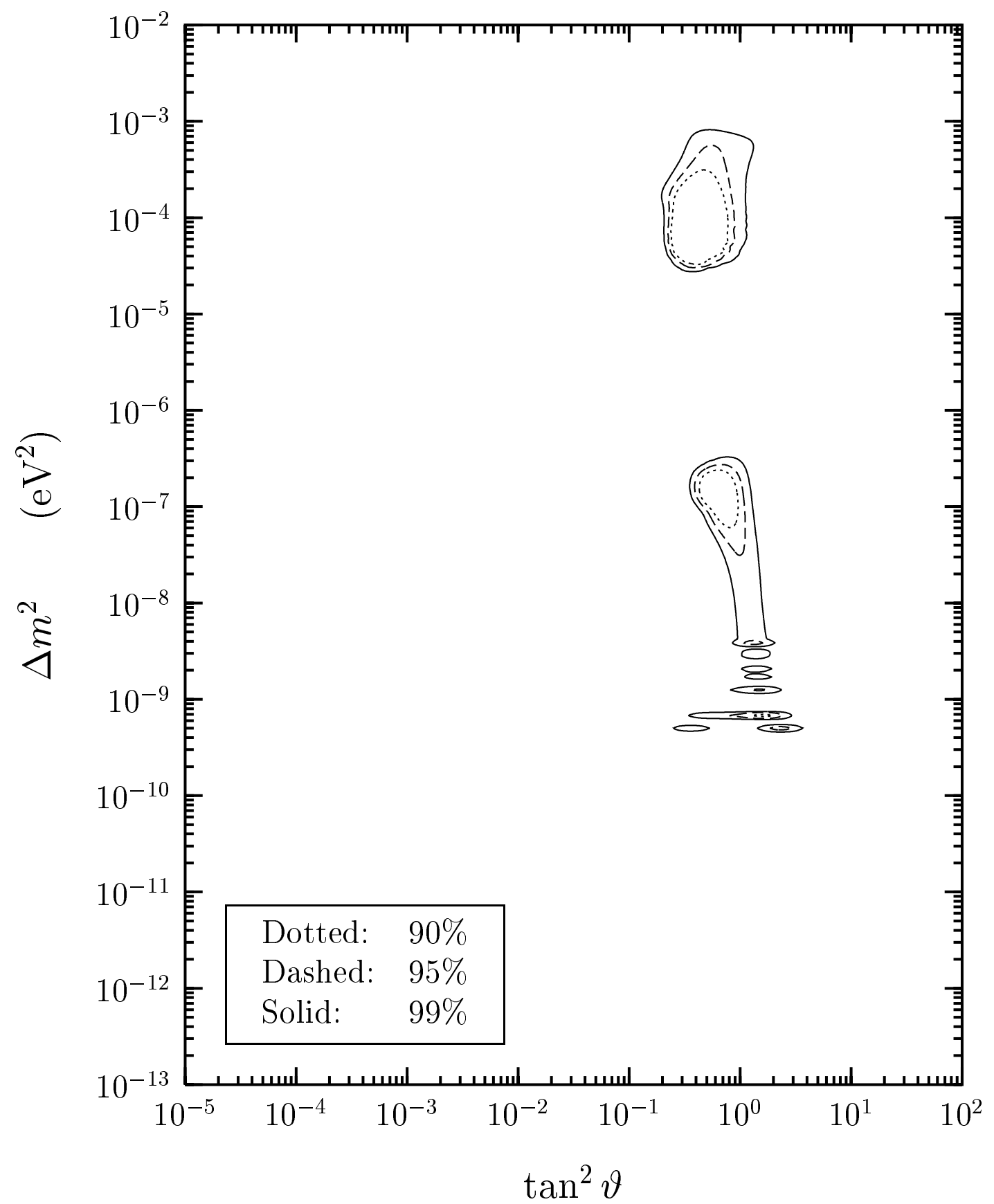

FIG. 2: Credible regions obtained with the Global Analysis of Homestake, GALLEX+GNO+SAGE and SNO rates and Super-Kamiokande day and night spectra, assuming the BP2000 Standard Solar Model neutrino fluxes. 


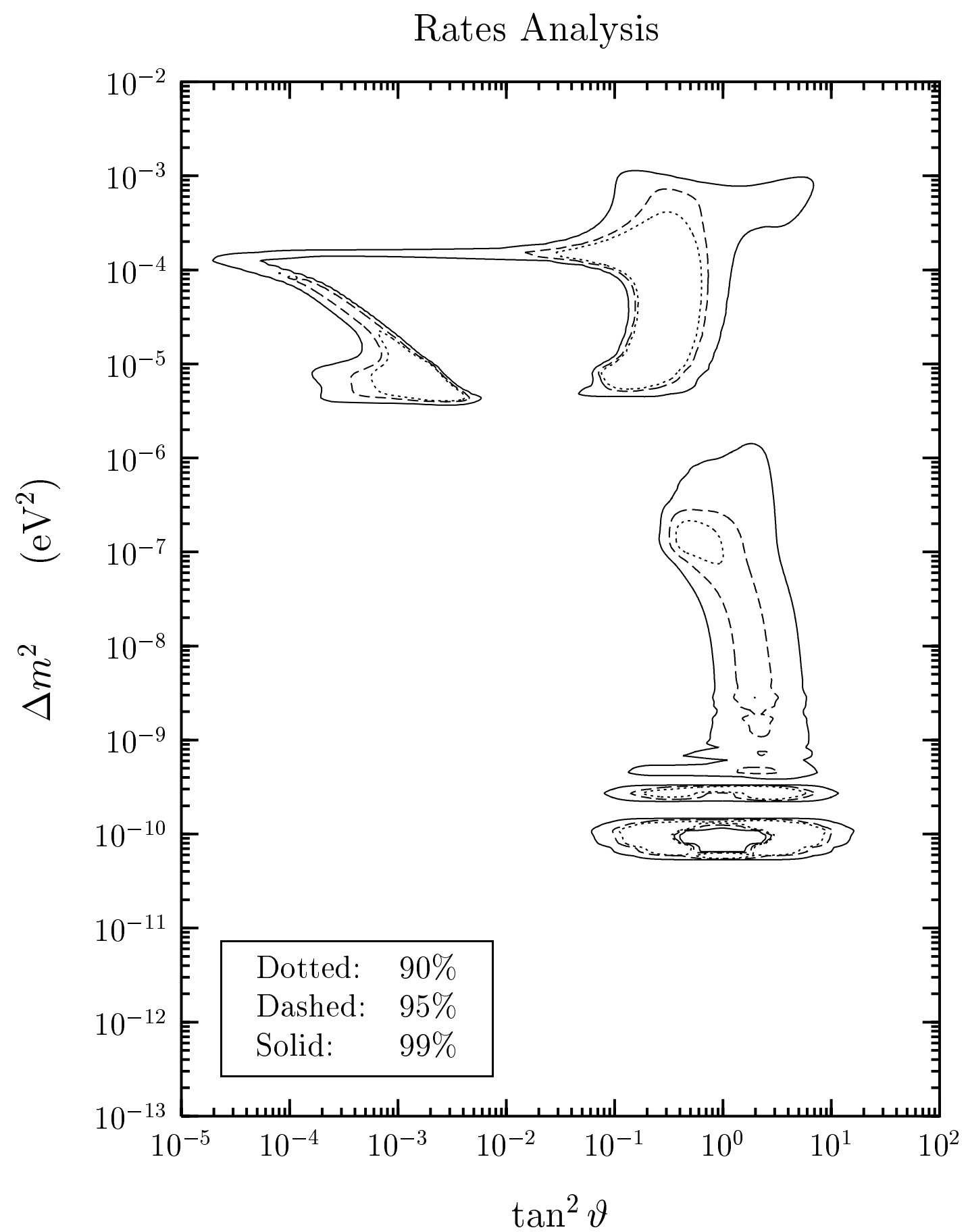

FIG. 3: Credible regions obtained with the model independent Rates Analysis of Homestake, GALLEX+GNO+SAGE, SNO and Super-Kamiokande total rates. 


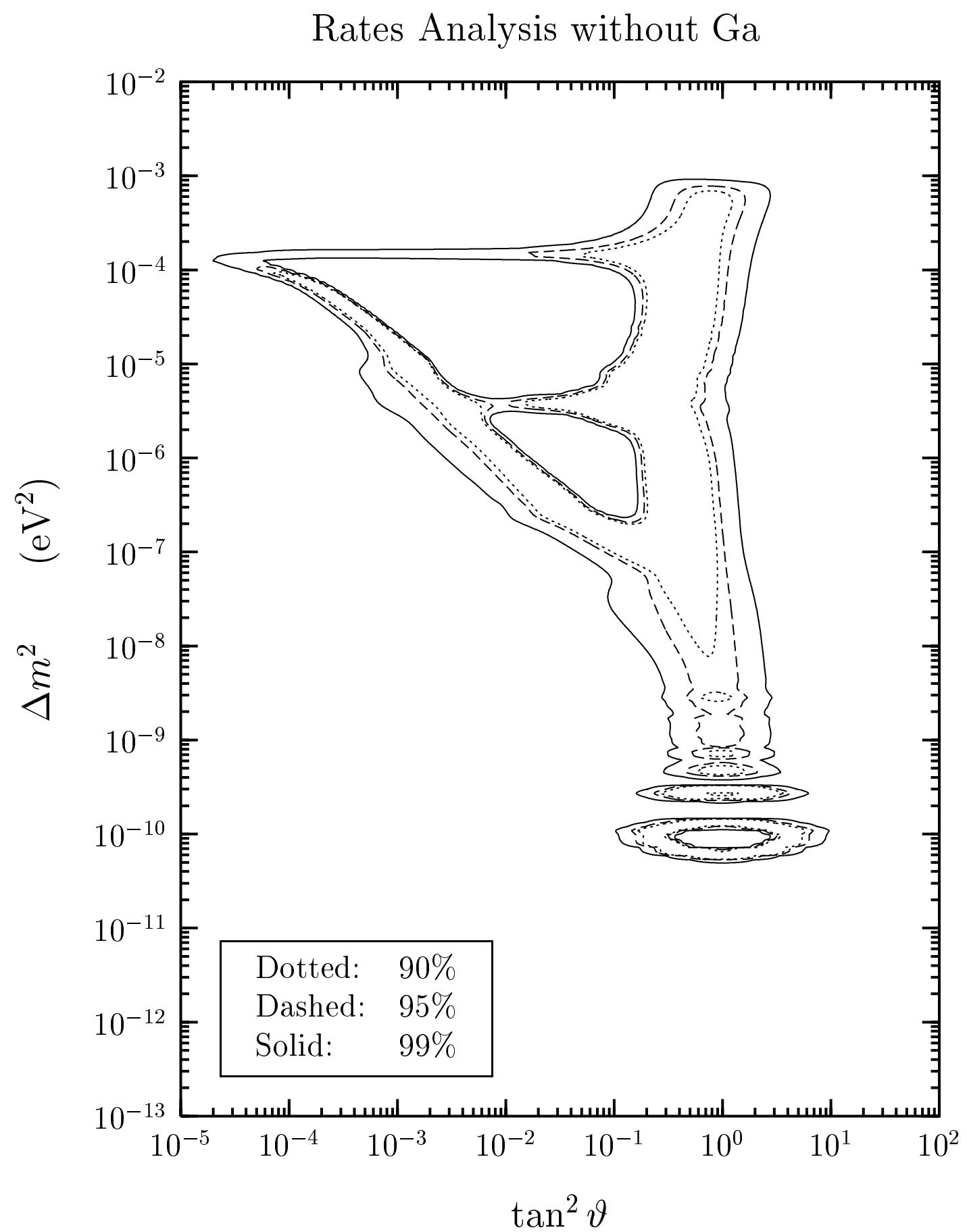

FIG. 4: Credible regions obtained with the model independent Rates Analysis of Homestake, SNO and Super-Kamiokande data, neglecting the Gallium data of the GALLEX, GNO and SAGE experiment. 


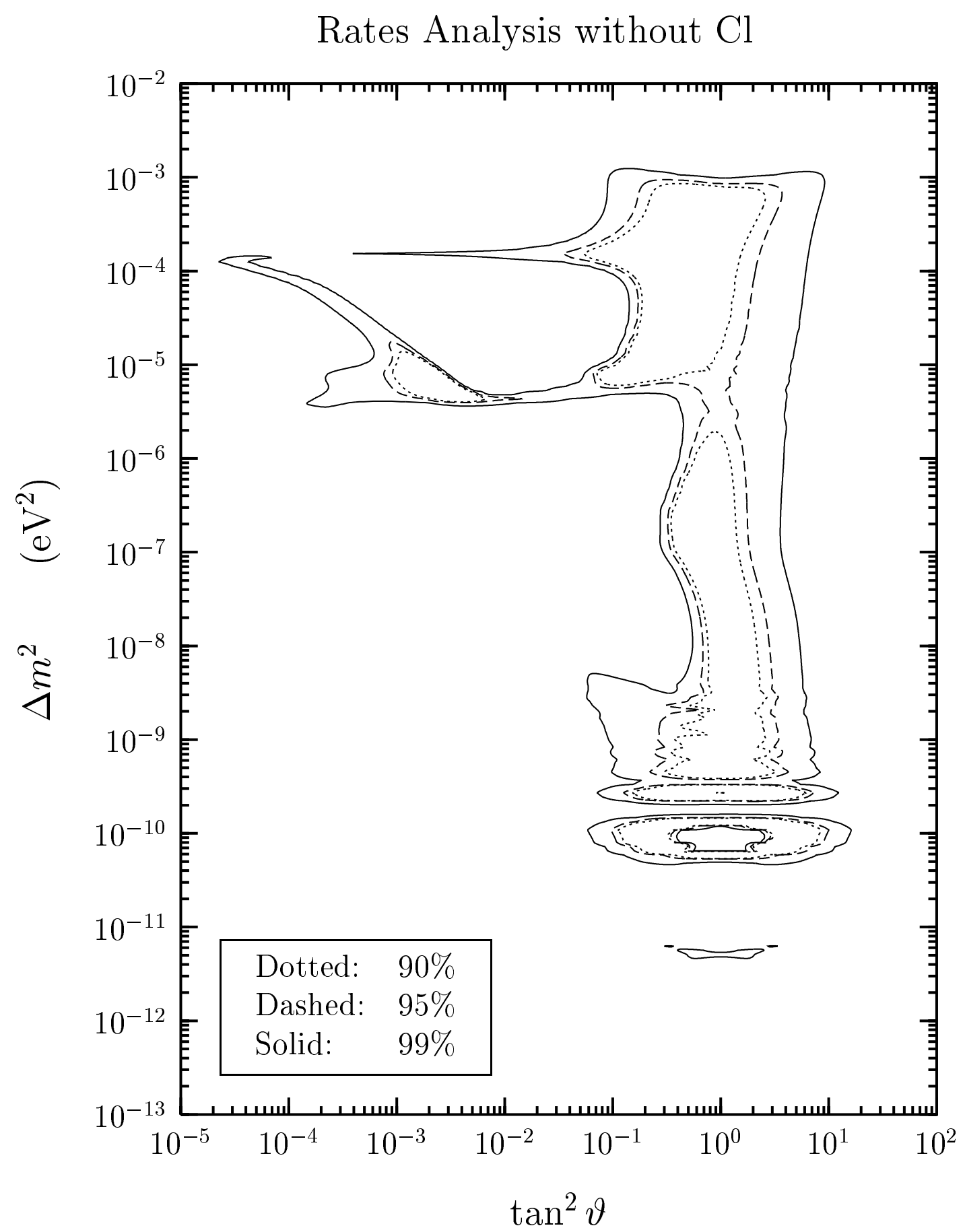

FIG. 5: Credible regions obtained with the model independent Rates Analysis of GALLEX+GNO+SAGE, SNO and SuperKamiokande data, neglecting the Chlorine data of the Homestake experiment. 


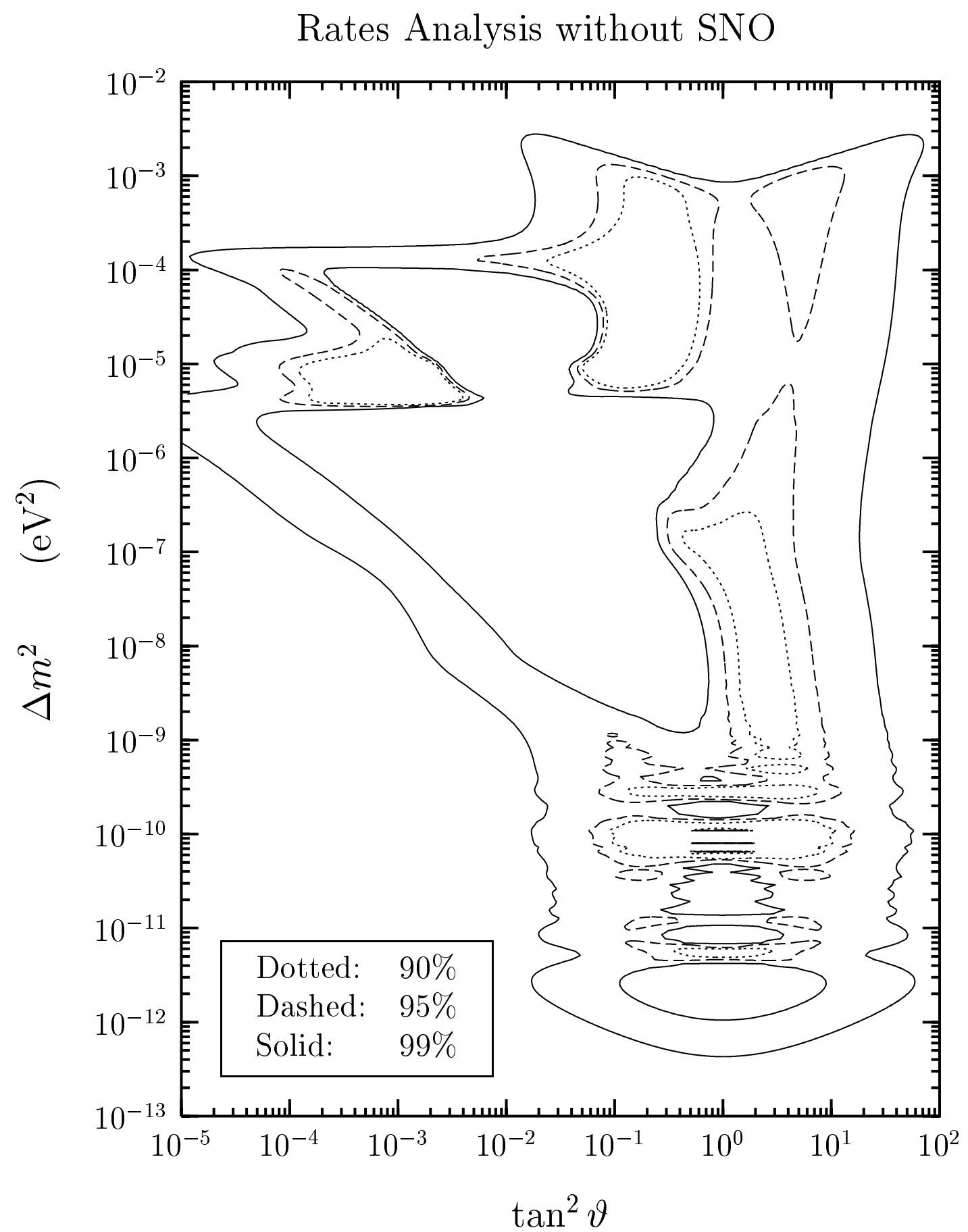

FIG. 6: Credible regions obtained with the model independent Rates Analysis of Homestake, GALLEX+GNO+SAGE and Super-Kamiokande data, neglecting the rate measured in the SNO experiment. 


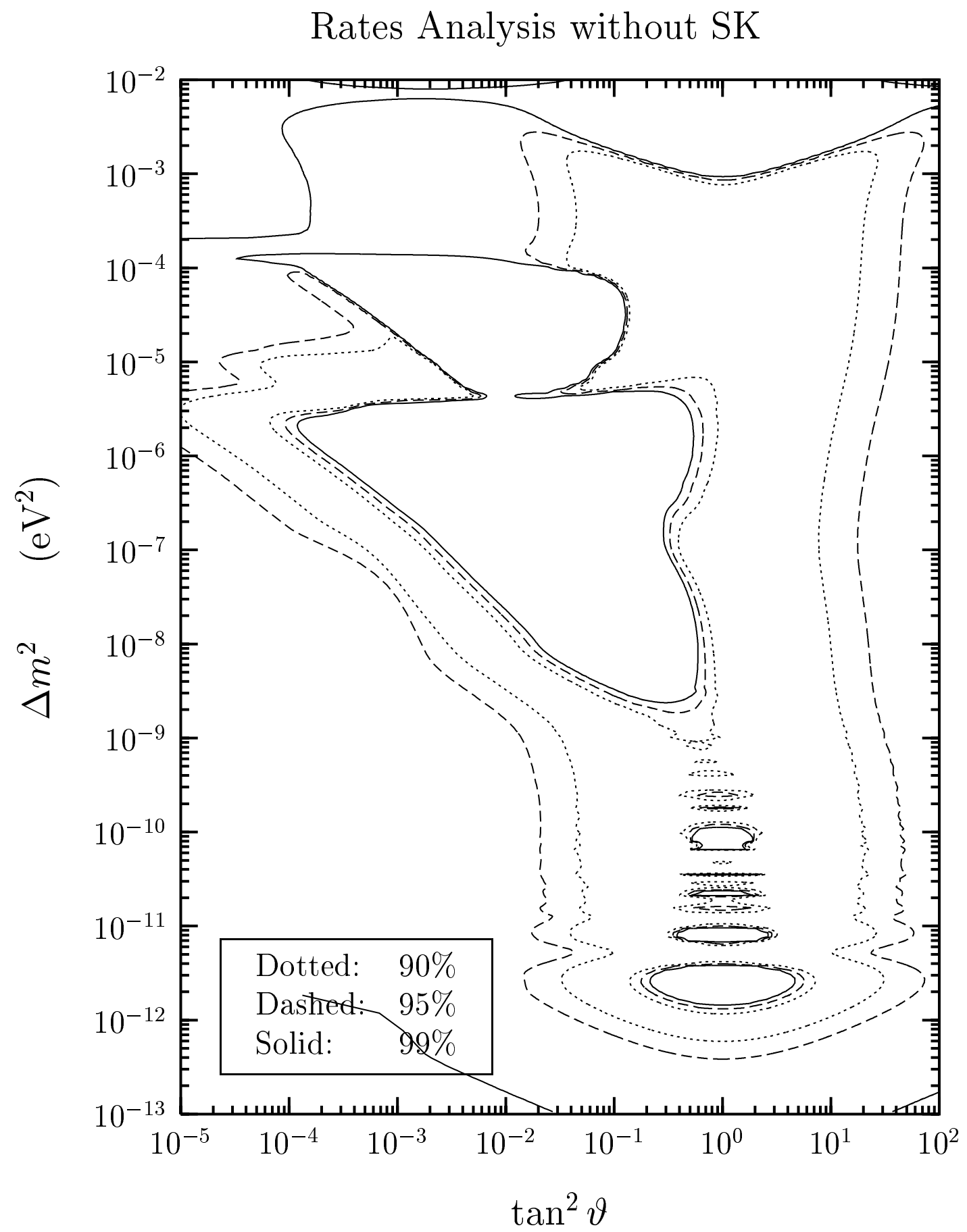

FIG. 7: Credible regions obtained with the model independent Rates Analysis of Homestake, GALLEX+GNO+SAGE and SNO data, neglecting the rate measured in the Super-Kamiokande experiment. 

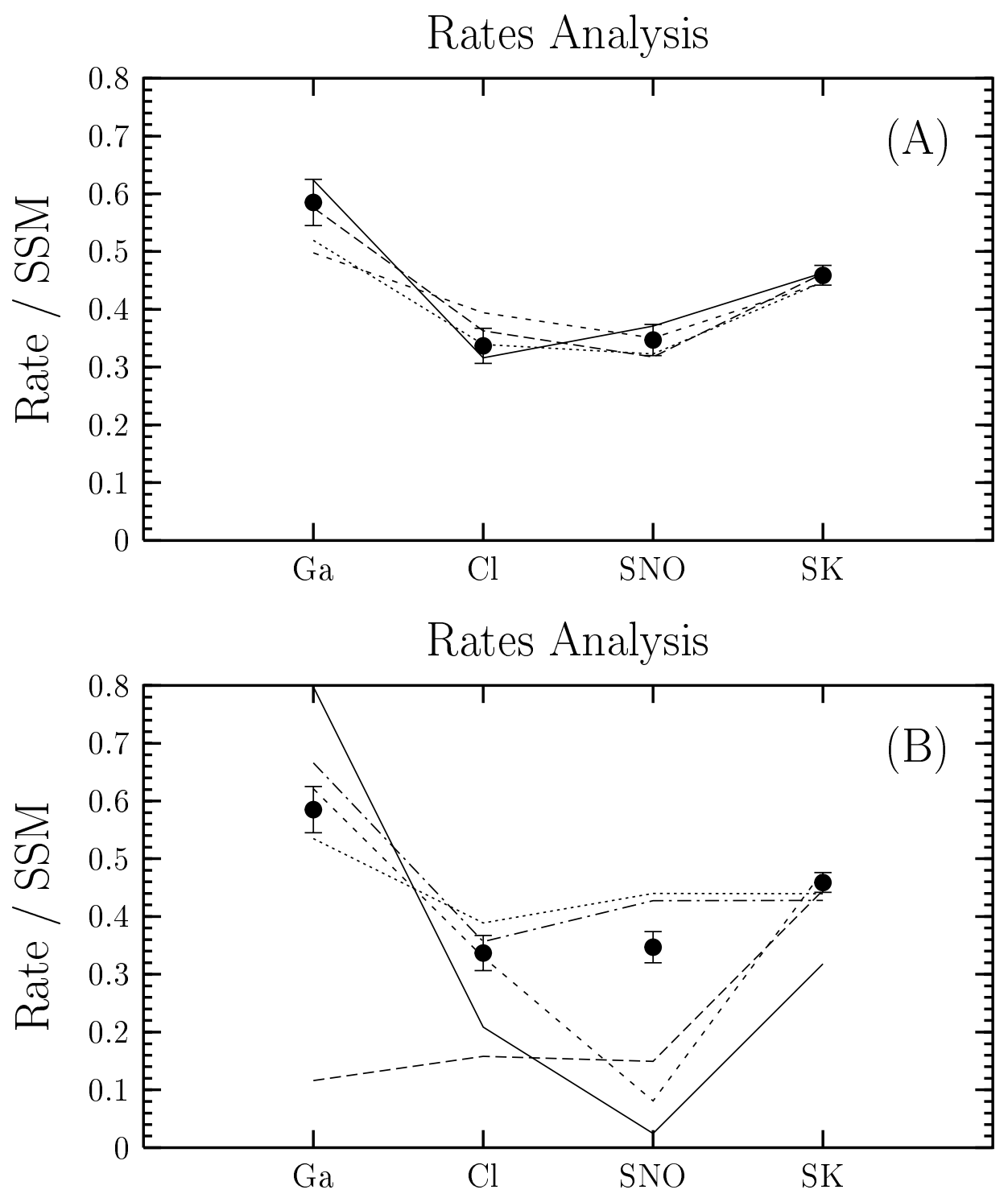

FIG. 8: Experimental rates (points with errorbars) and theoretical predictions for nine selected values of $\tan ^{2} \vartheta$ and $\Delta m^{2}$ in the Rates Analysis. (A). Solid line: $\tan ^{2} \vartheta=1.7 \times 10^{-3}$ and $\Delta m^{2}=8.0 \times 10^{-6} \mathrm{eV}^{2}$, best fit point in the SMA region in the Rates Analysis; long-dashed line: $\tan ^{2} \vartheta=0.20$ and $\Delta m^{2}=1.2 \times 10^{-5} \mathrm{eV}^{2}$, best fit point in the LMA region in the Rates Analysis; short-dashed line: $\tan ^{2} \vartheta=0.49$ and $\Delta m^{2}=1.4 \times 10^{-7} \mathrm{eV}^{2}$, best fit point in the LOW region in the Rates Analysis; dotted line: $\tan ^{2} \vartheta=3.59$ and $\Delta m^{2}=8.0 \times 10^{-11} \mathrm{eV}^{2}$, best fit point in the VO region in the Rates Analysis. (B). Solid line: $\tan ^{2} \vartheta=1.0 \times 10^{-2}$ and $\Delta m^{2}=3.0 \times 10^{-5} \mathrm{eV}^{2}$; long-dashed line: $\tan ^{2} \vartheta=5.0 \times 10^{-2}$ and $\Delta m^{2}=1.0 \times 10^{-6} \mathrm{eV}^{2} ;$ short-dashed line: $\tan ^{2} \vartheta=1.00$ and $\Delta m^{2}=9.0 \times 10^{-11} \mathrm{eV}^{2}$; dotted line: $\tan ^{2} \vartheta=1.00$ and $\Delta m^{2}=5.5 \times 10^{-12} \mathrm{eV}^{2}$; dash-dotted line: no oscillations. 


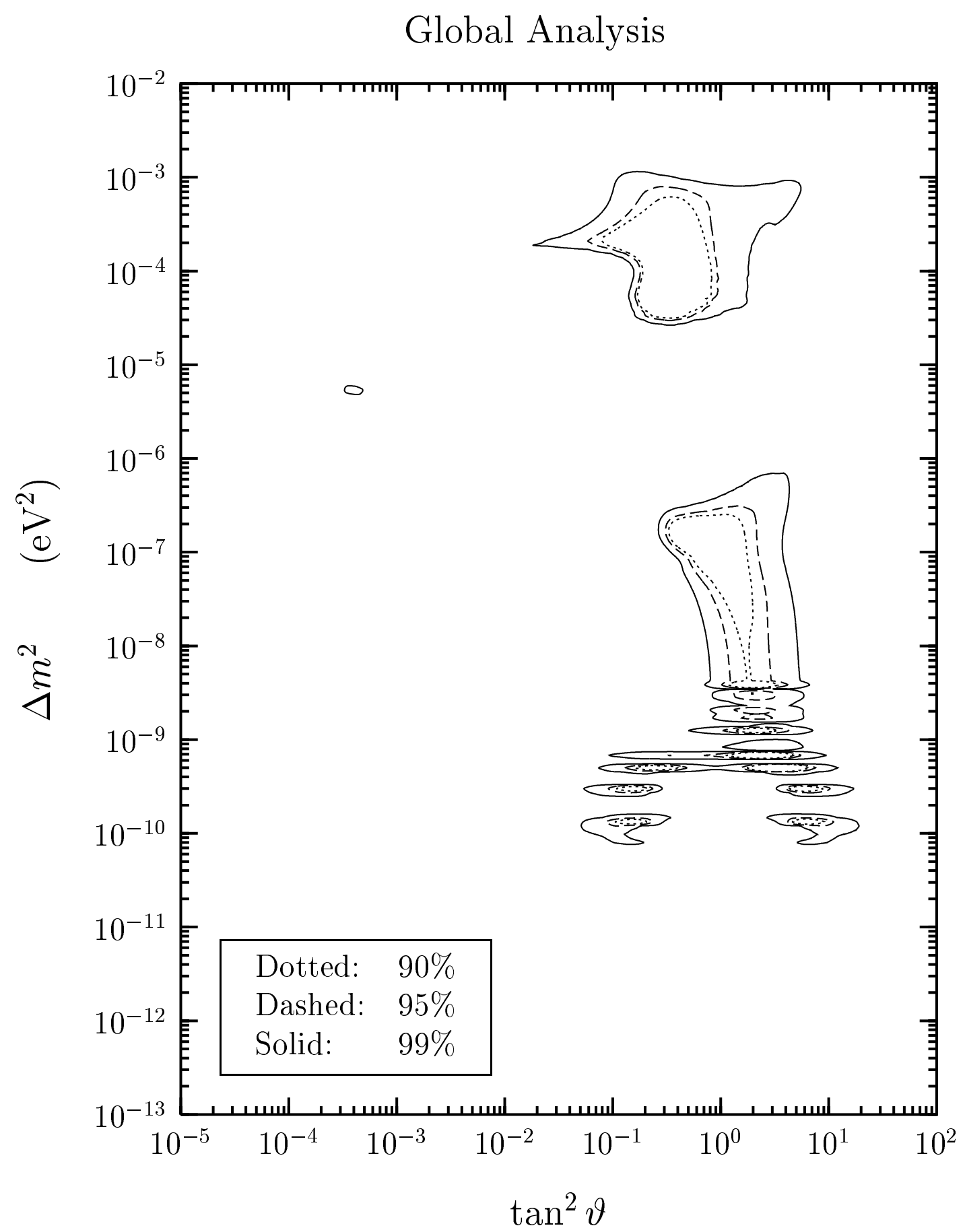

FIG. 9: Credible regions obtained with the model independent Global Analysis of Homestake, GALLEX+GNO+SAGE and SNO rates and Super-Kamiokande day and night spectra. 

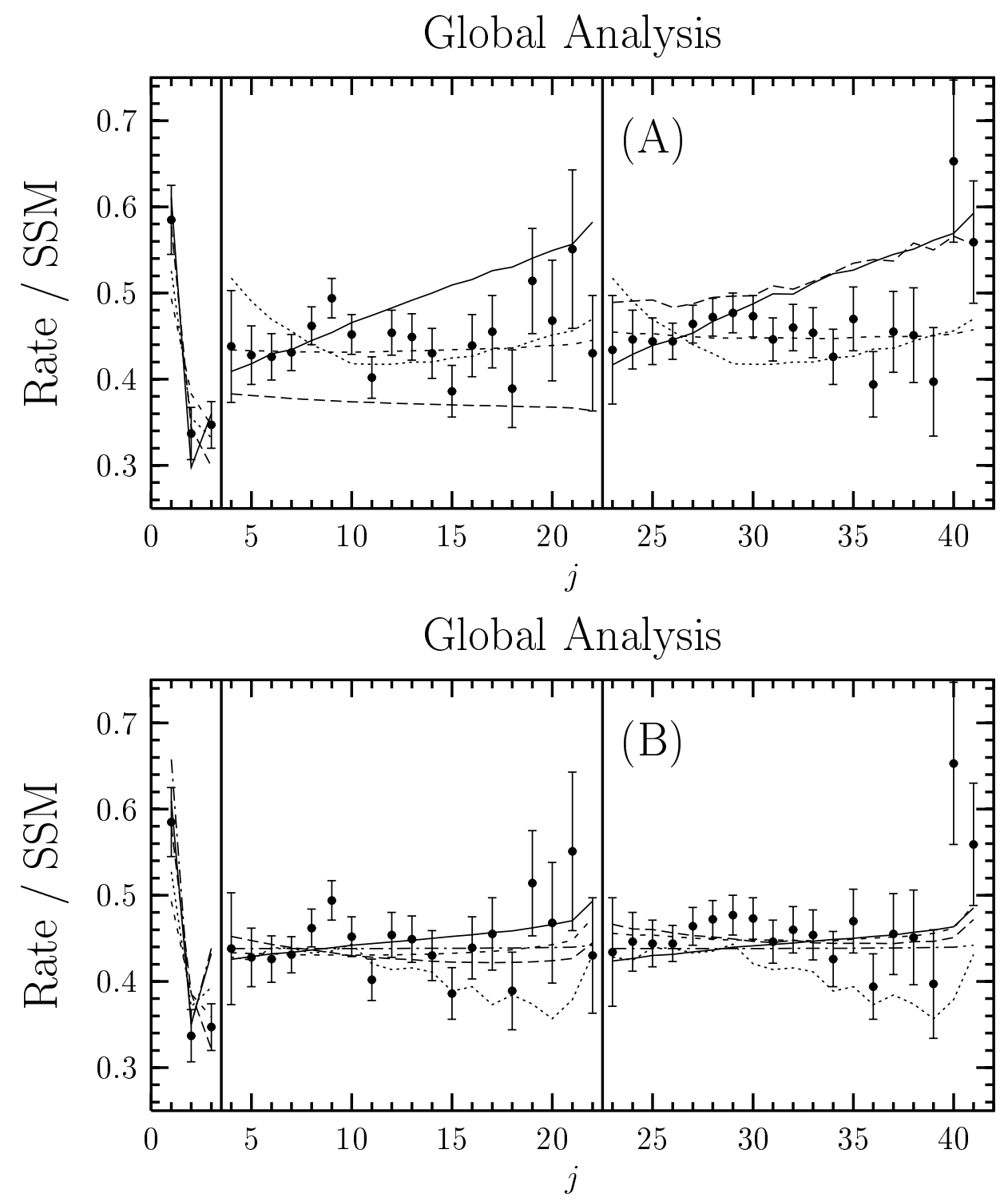

FIG. 10: Experimental rates (points with errorbars) and theoretical predictions for nine selected values of tan ${ }^{2} \vartheta$ and $\Delta m^{2}$ in the Global Analysis. The index $j$ labels the solar data points: $j=1,2,3$ for the Gallium, Chlorine and SNO event rates, respectively; $j=4, \ldots, 22$ for the Super-Kamiokande energy spectrum in the day; $j=23, \ldots, 41$ for the Super-Kamiokande energy spectrum in the night. (A). Solid line: $\tan ^{2} \vartheta=1.7 \times 10^{-3}$ and $\Delta m^{2}=8.0 \times 10^{-6} \mathrm{eV}^{2}$, best fit point in the SMA region in the Rates Analysis; long-dashed line: $\tan ^{2} \vartheta=0.20$ and $\Delta m^{2}=1.2 \times 10^{-5} \mathrm{eV}^{2}$, best fit point in the LMA region in the Rates Analysis; short-dashed line: $\tan ^{2} \vartheta=0.49$ and $\Delta m^{2}=1.4 \times 10^{-7} \mathrm{eV}^{2}$, best fit point in the LOW region in the Rates Analysis; dotted line: $\tan ^{2} \vartheta=3.59$ and $\Delta m^{2}=8.0 \times 10^{-11} \mathrm{eV}^{2}$, best fit point in the VO region in the Rates Analysis. (B). Solid line: $\tan ^{2} \vartheta=3.8 \times 10^{-4}$ and $\Delta m^{2}=5.3 \times 10^{-6} \mathrm{eV}^{2}$, best fit point in the SMA region in the Global Analysis; long-dashed line: $\tan ^{2} \vartheta=0.34$ and $\Delta m^{2}=6.1 \times 10^{-5} \mathrm{eV}^{2}$, best fit point in the LMA region in the Global Analysis; short-dashed line: $\tan ^{2} \vartheta=0.60$ and $\Delta m^{2}=1.5 \times 10^{-7} \mathrm{eV}^{2}$, best fit point in the LOW region in the Global Analysis; dotted line: $\tan ^{2} \vartheta=3.16$ and $\Delta m^{2}=5.0 \times 10^{-10} \mathrm{eV}^{2}$, best fit point in the VO region in the Global Analysis; dash-dotted line: no oscillations. 


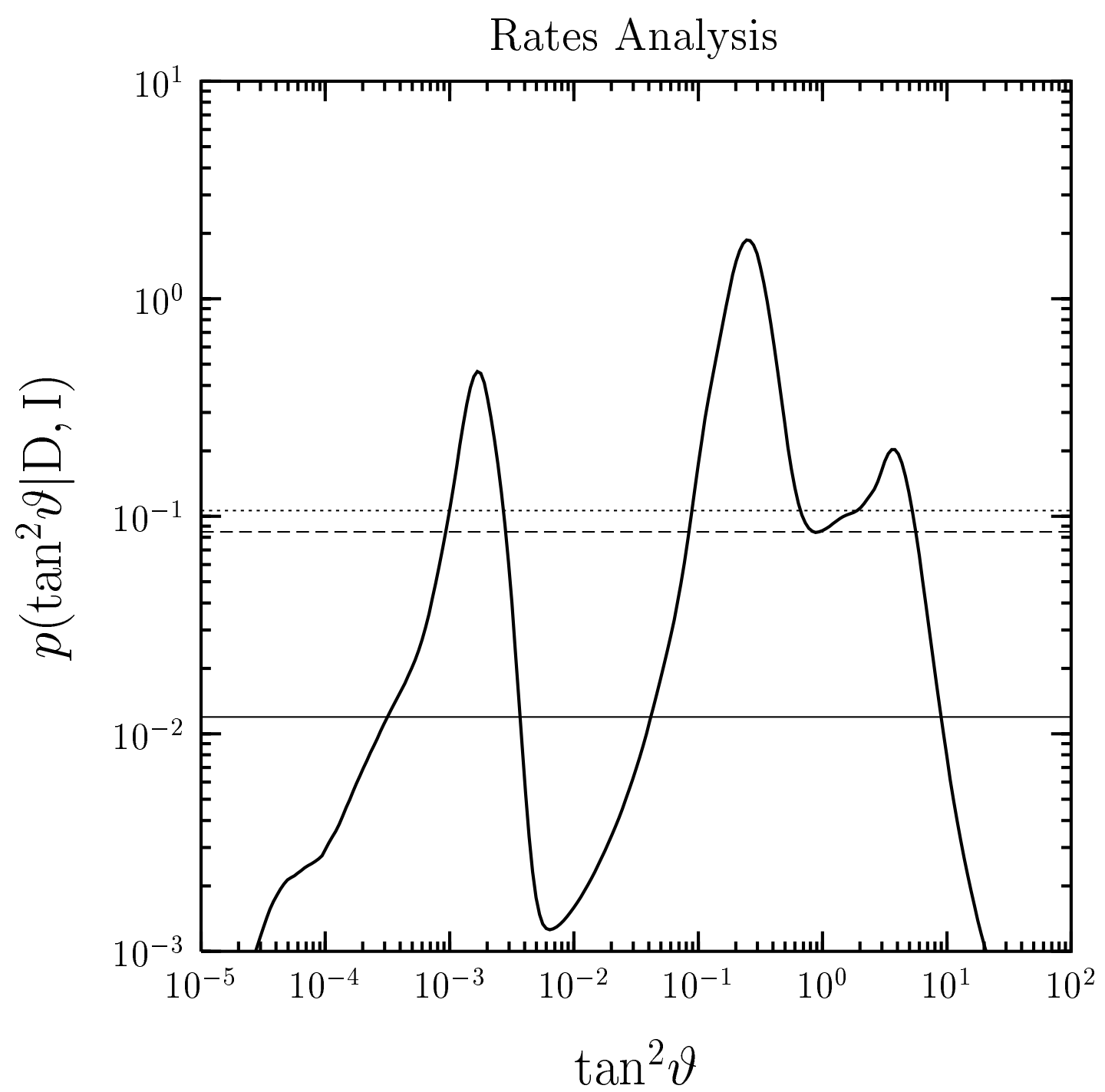

FIG. 11: Posterior distribution of $\tan ^{2} \vartheta$ obtained with the model independent Rates Analysis. The intervals in which the thick solid curve lies above the horizontal dotted, dashed, and solid lines have, respectively, $90 \%, 95 \%$ and $99 \%$ posterior probability to contain the true value of $\tan ^{2} \vartheta$. 


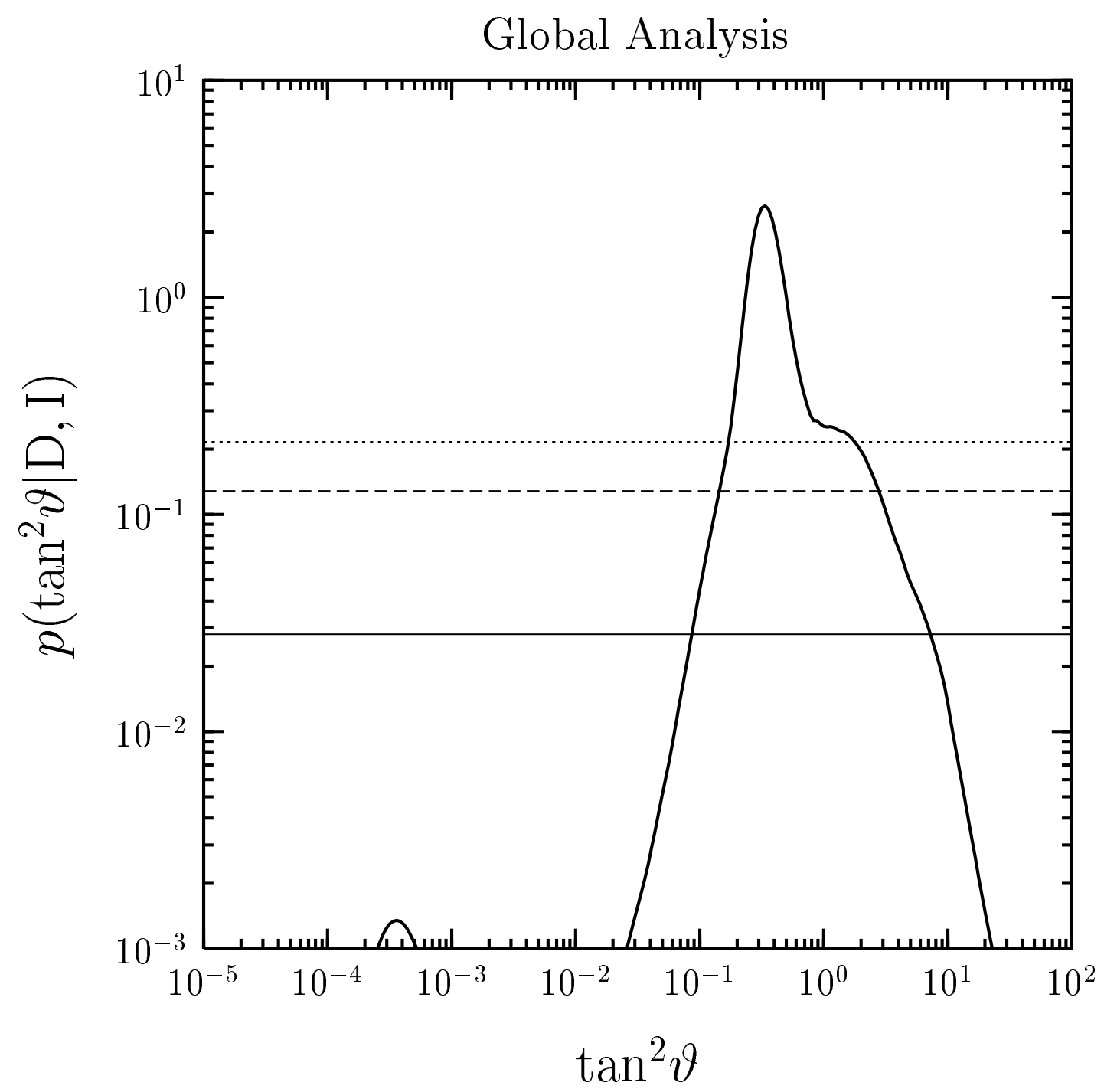

FIG. 12: Posterior distribution of $\tan ^{2} \vartheta$ obtained with the model independent Global Analysis. The intervals in which the thick solid curve lies above the horizontal dotted, dashed, and solid lines have, respectively, 90\%, 95\% and 99\% posterior probability to contain the true value of $\tan ^{2} \vartheta$. 


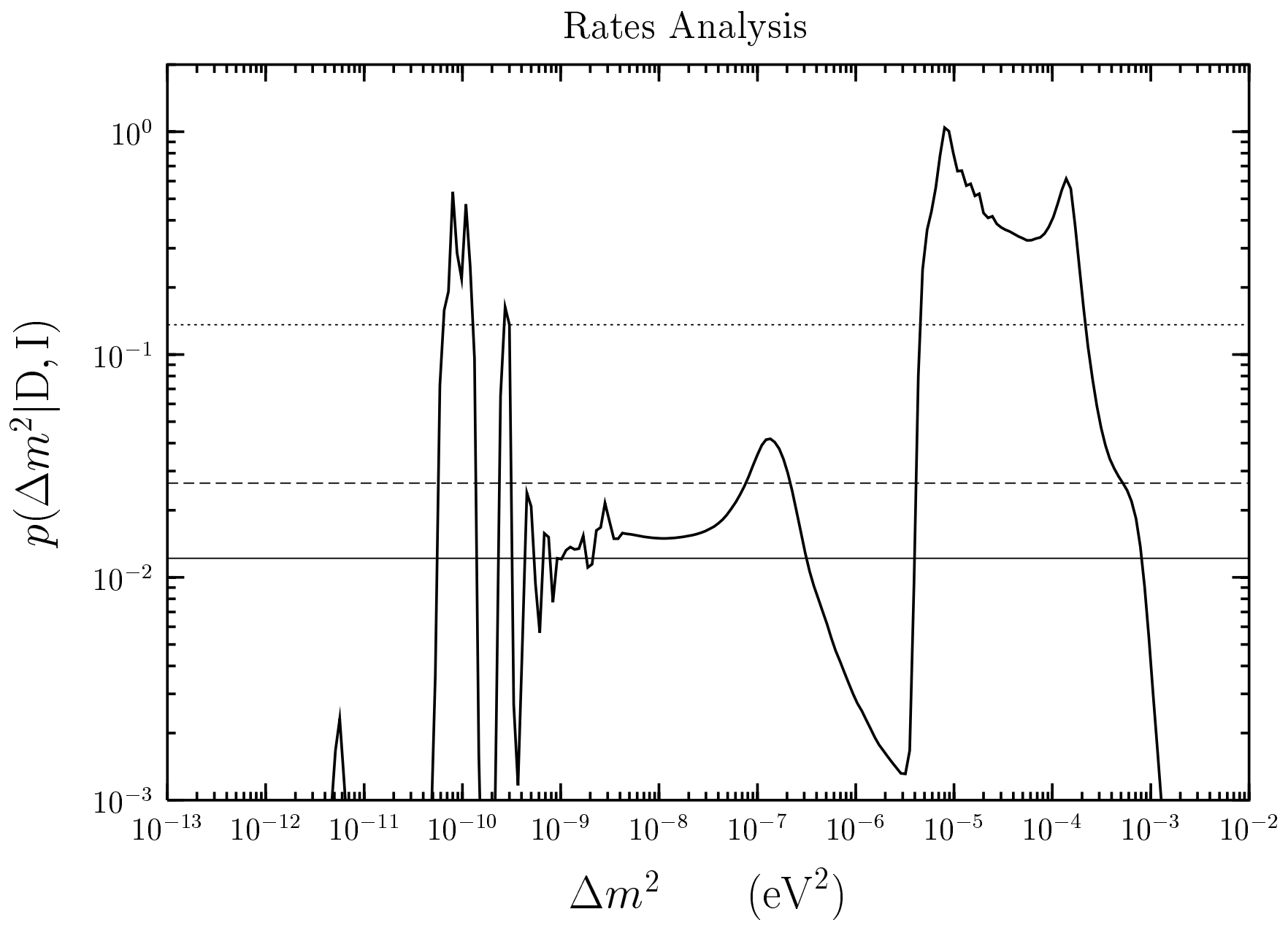

FIG. 13: Posterior distribution of $\Delta m^{2}$ obtained with the model independent Rates Analysis. The intervals in which the thick solid curve lies above the horizontal dotted, dashed, and solid lines have, respectively, 90\%, 95\% and 99\% posterior probability to contain the true value of $\Delta m^{2}$. 


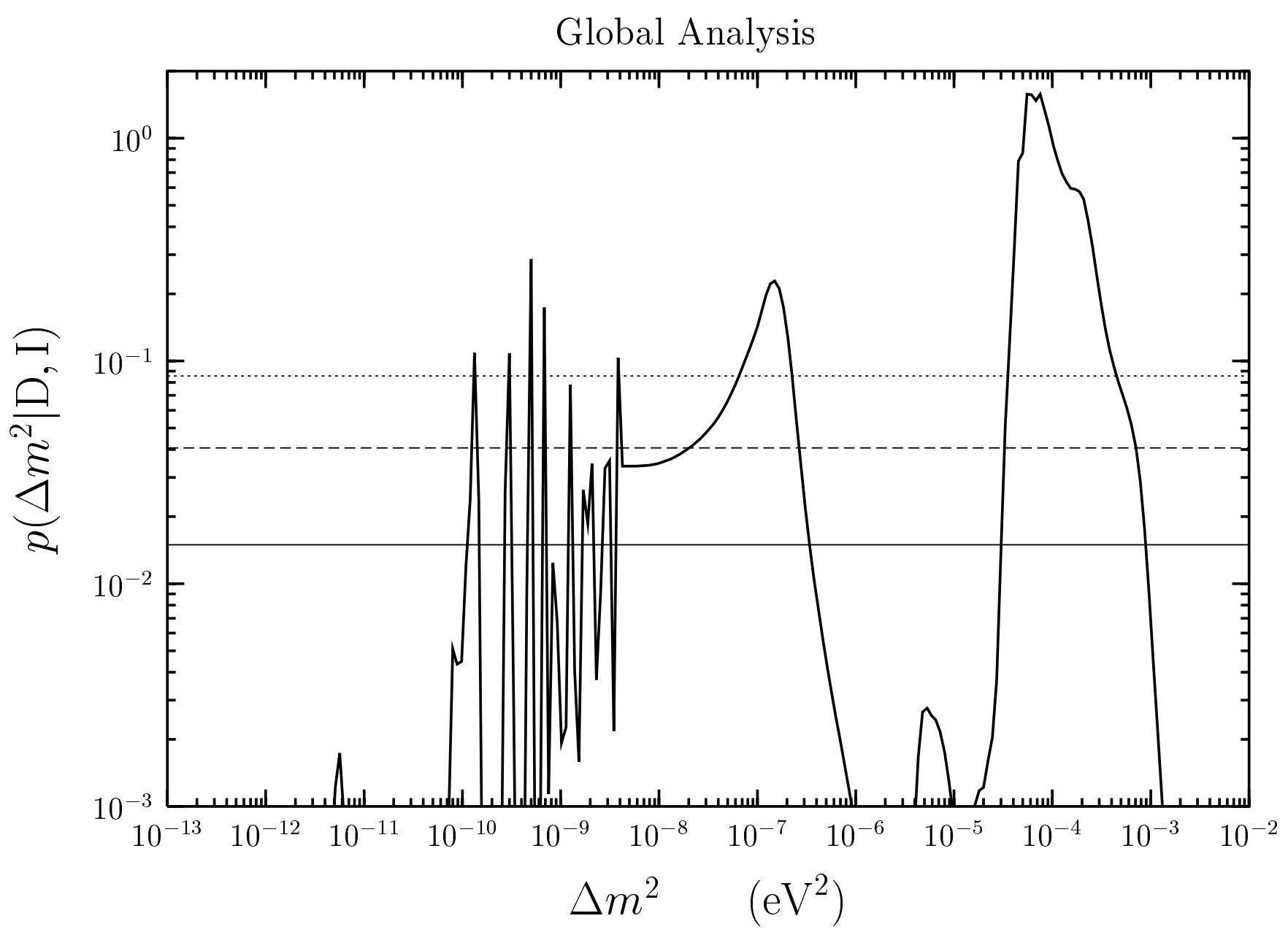

FIG. 14: Posterior distribution of $\Delta m^{2}$ obtained with the model independent Global Analysis. The intervals in which the thick solid curve lies above the horizontal dotted, dashed, and solid lines have, respectively, 90\%, 95\% and 99\% posterior probability to contain the true value of $\Delta m^{2}$. 


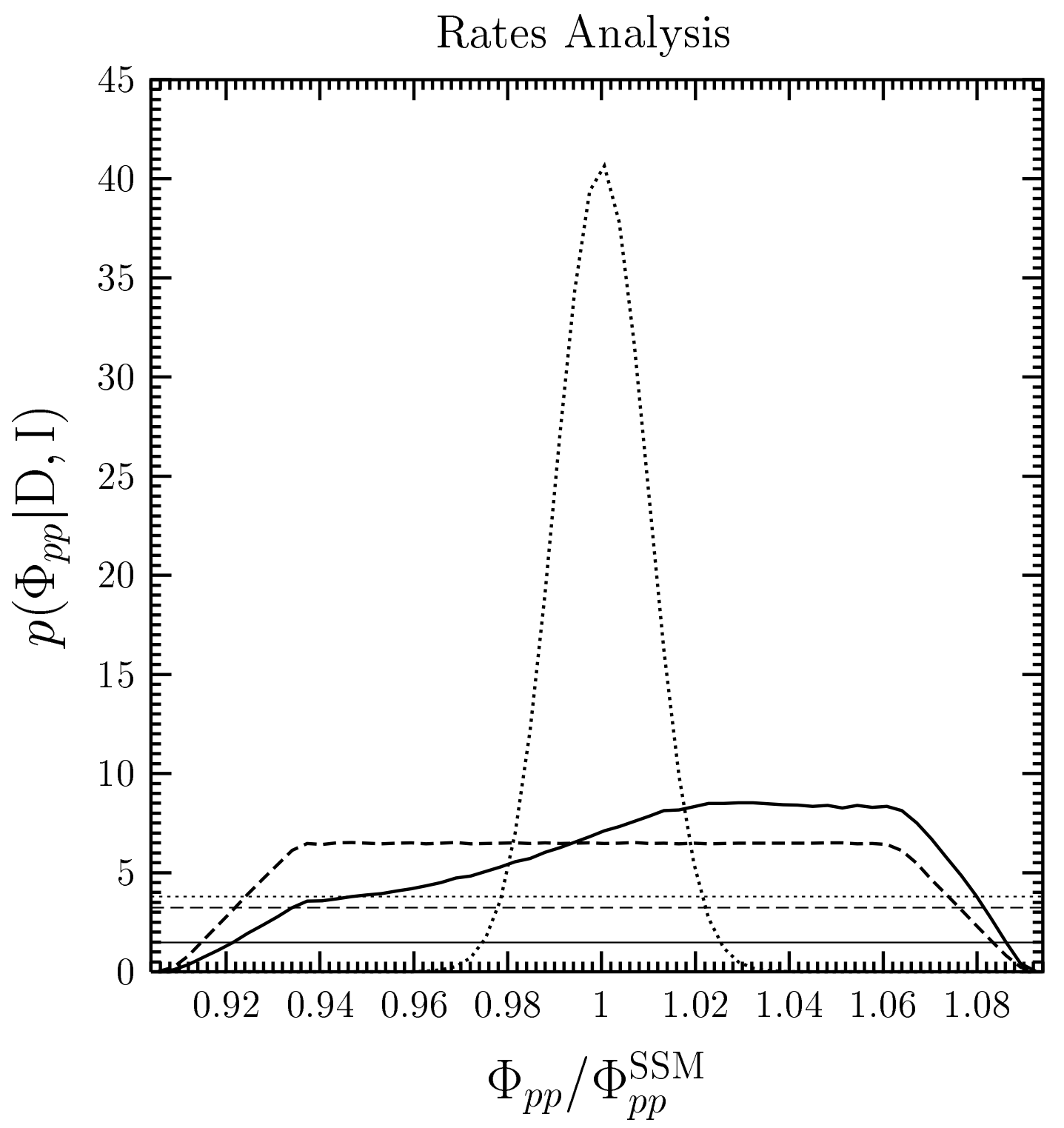

FIG. 15: The thick solid line is the posterior distribution of the $p p$ flux obtained with the model independent Rates Analysis. The intervals in which the thick solid line lies above the horizontal thin dotted, dashed, and solid lines have, respectively, $90 \%$, $95 \%$ and $99 \%$ posterior probability to contain the true value of the $p p$ flux. The gaussian thick dotted curve is the BP2000 SSM distribution and the thick dashed curve is the prior distribution in the model independent analysis. 


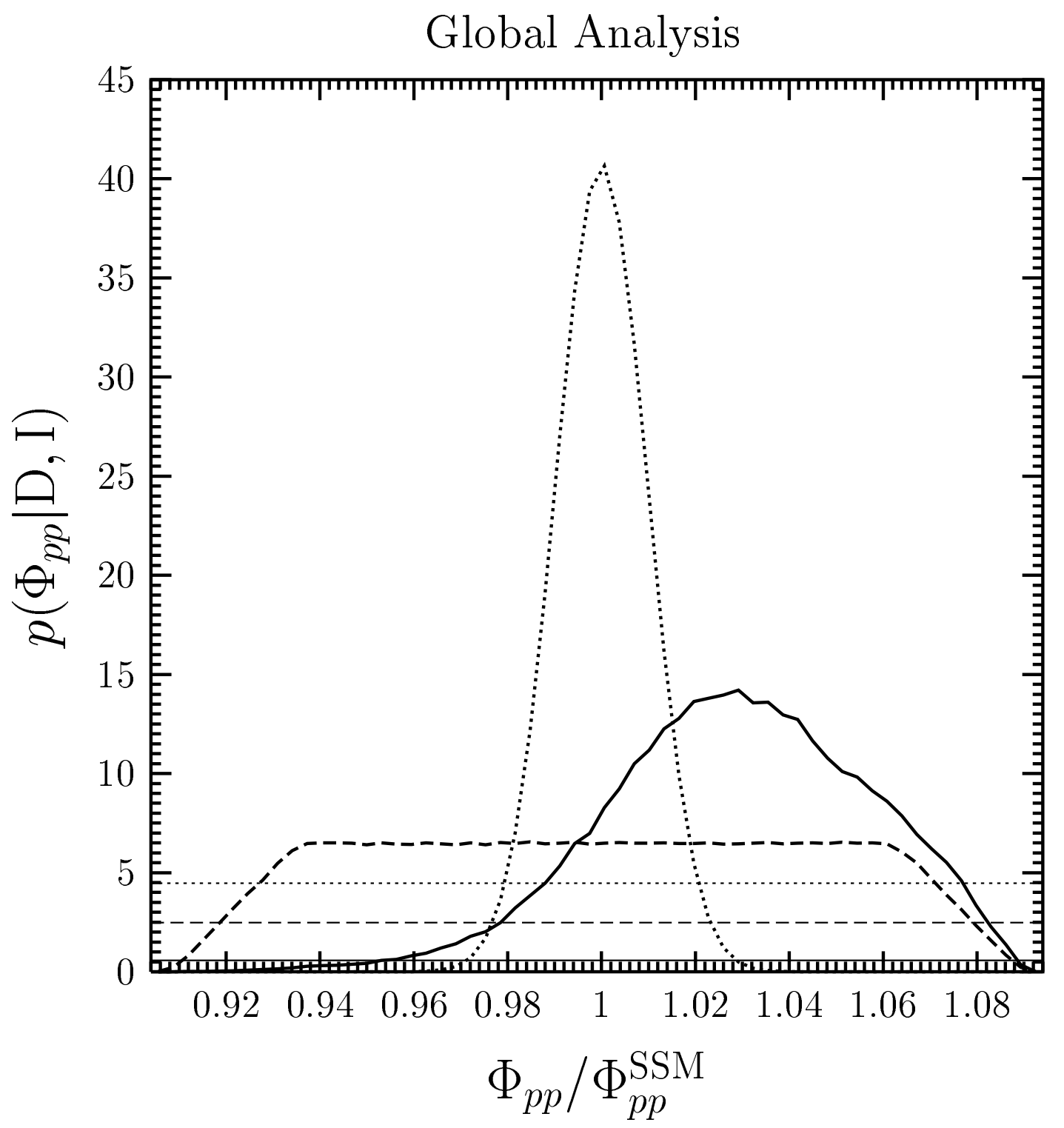

FIG. 16: The thick solid line is the posterior distribution of the $p p$ flux obtained with the model independent Global Analysis. The intervals in which the thick solid line lies above the horizontal thin dotted, dashed, and solid lines have, respectively, $90 \%$, $95 \%$ and $99 \%$ posterior probability to contain the true value of the $p p$ flux. The gaussian thick dotted curve is the BP2000 SSM distribution and the thick dashed curve is the prior distribution in the model independent analysis. 


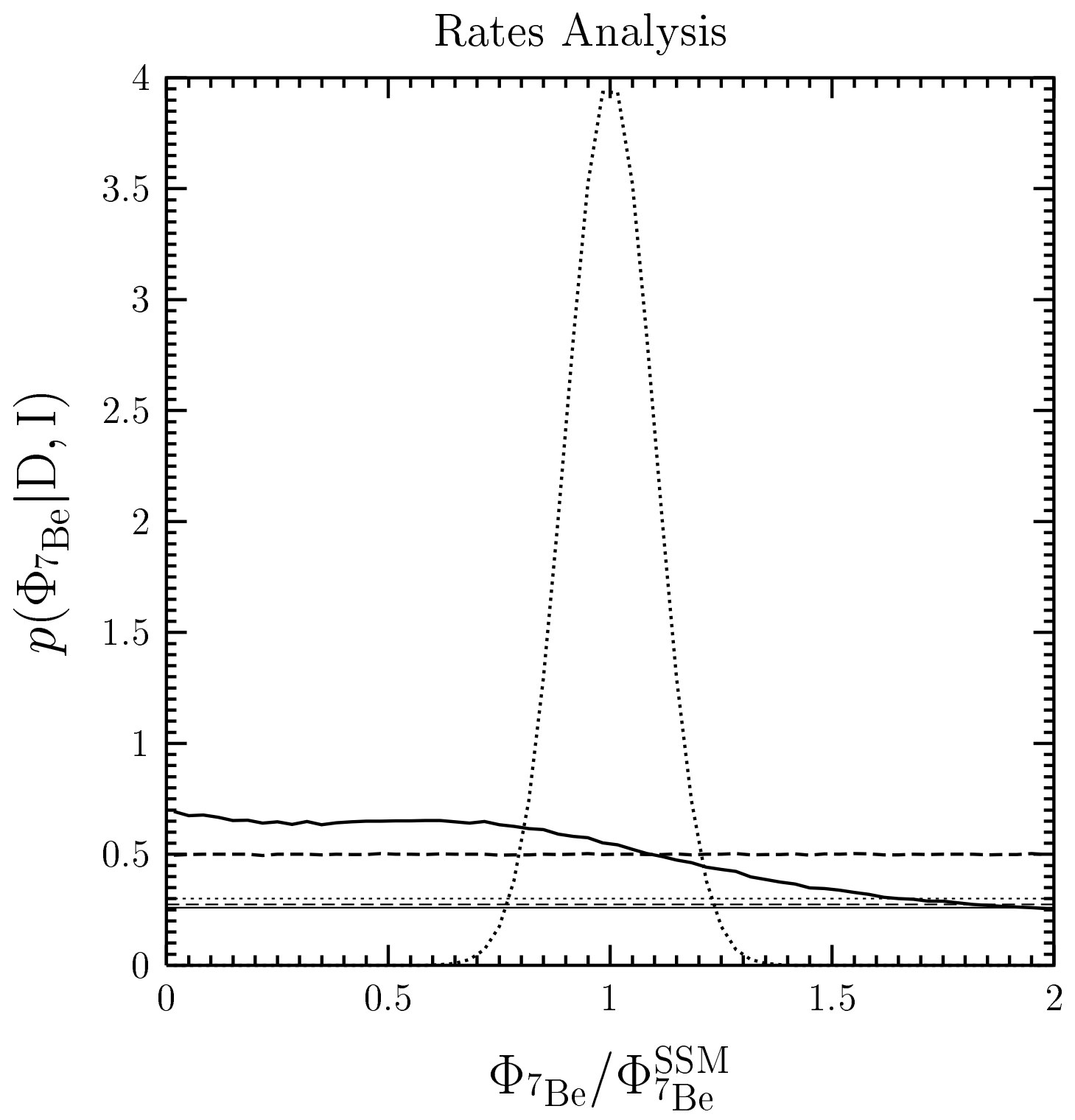

FIG. 17: The thick solid line is the posterior distribution of the ${ }^{7}$ Be flux obtained with the model independent Rates Analysis. The intervals in which the thick solid line lies above the horizontal thin dotted, dashed, and solid lines have, respectively, $90 \%$, $95 \%$ and $99 \%$ posterior probability to contain the true value of the $p p$ flux. The gaussian thick dotted curve is the BP2000 SSM distribution and the thick dashed curve is the prior distribution in the model independent analysis. 


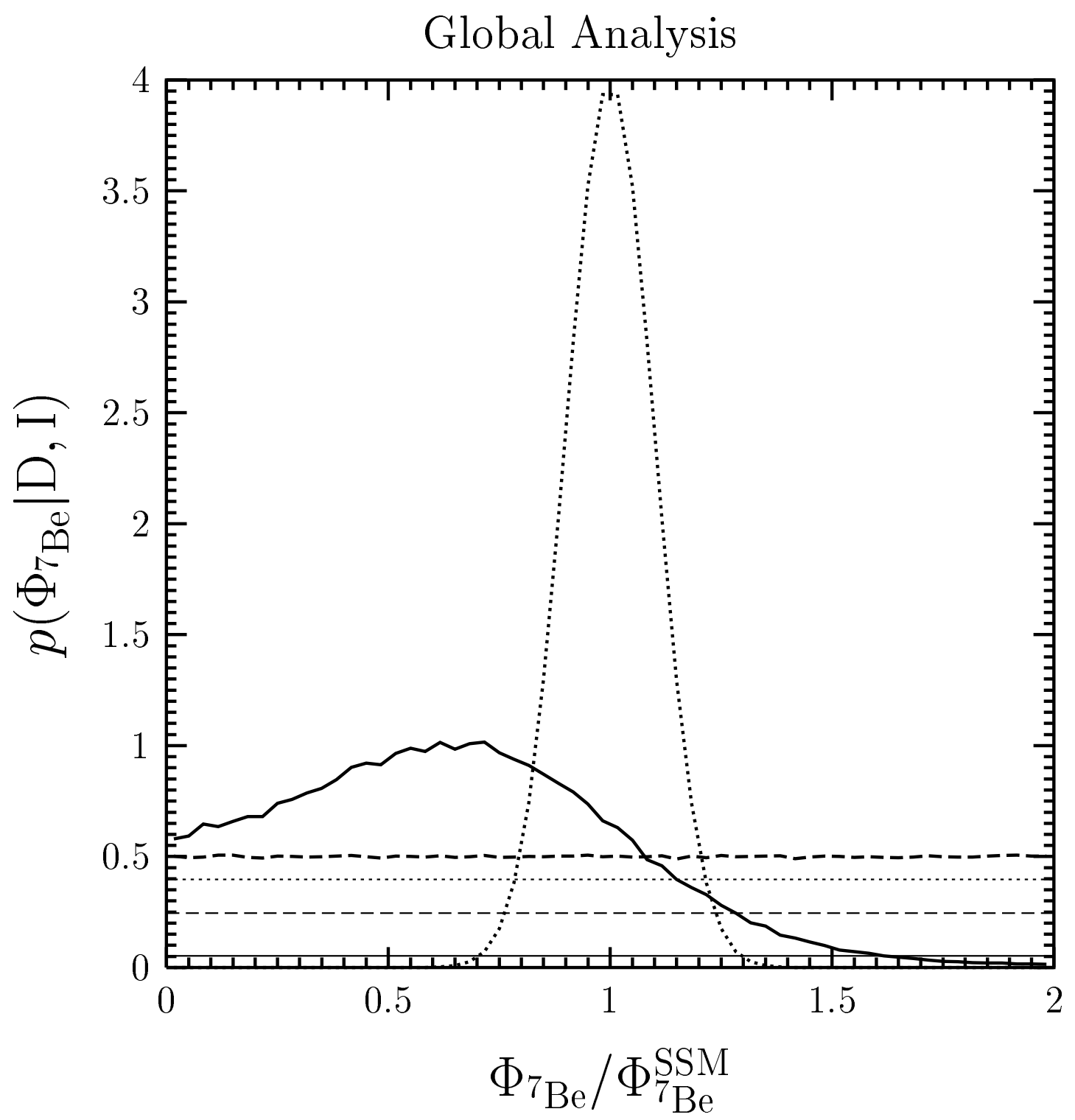

FIG. 18: The thick solid line is the posterior distribution of the ${ }^{7}$ Be flux obtained with the model independent Global Analysis. The intervals in which the thick solid line lies above the horizontal thin dotted, dashed, and solid lines have, respectively, $90 \%$, $95 \%$ and $99 \%$ posterior probability to contain the true value of the $p p$ flux. The gaussian thick dotted curve is the BP2000 SSM distribution and the thick dashed curve is the prior distribution in the model independent analysis. 


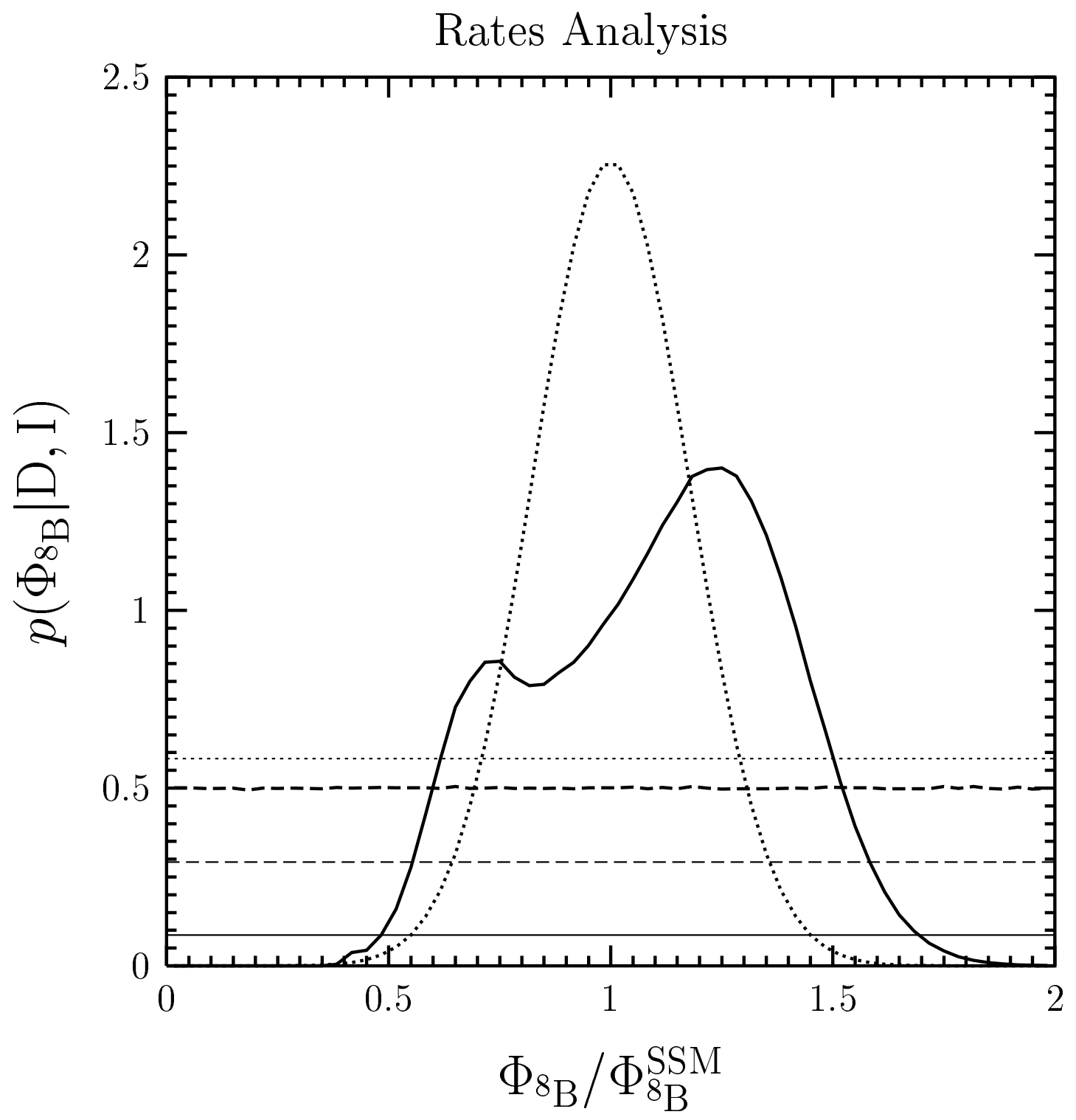

FIG. 19: The thick solid line is the posterior distribution of the ${ }^{8} \mathrm{~B}$ flux obtained with the model independent Rates Analysis. The intervals in which the thick solid line lies above the horizontal thin dotted, dashed, and solid lines have, respectively, $90 \%$, $95 \%$ and $99 \%$ posterior probability to contain the true value of the $p p$ flux. The gaussian thick dotted curve is the BP2000 SSM distribution and the thick dashed curve is the prior distribution in the model independent analysis. 


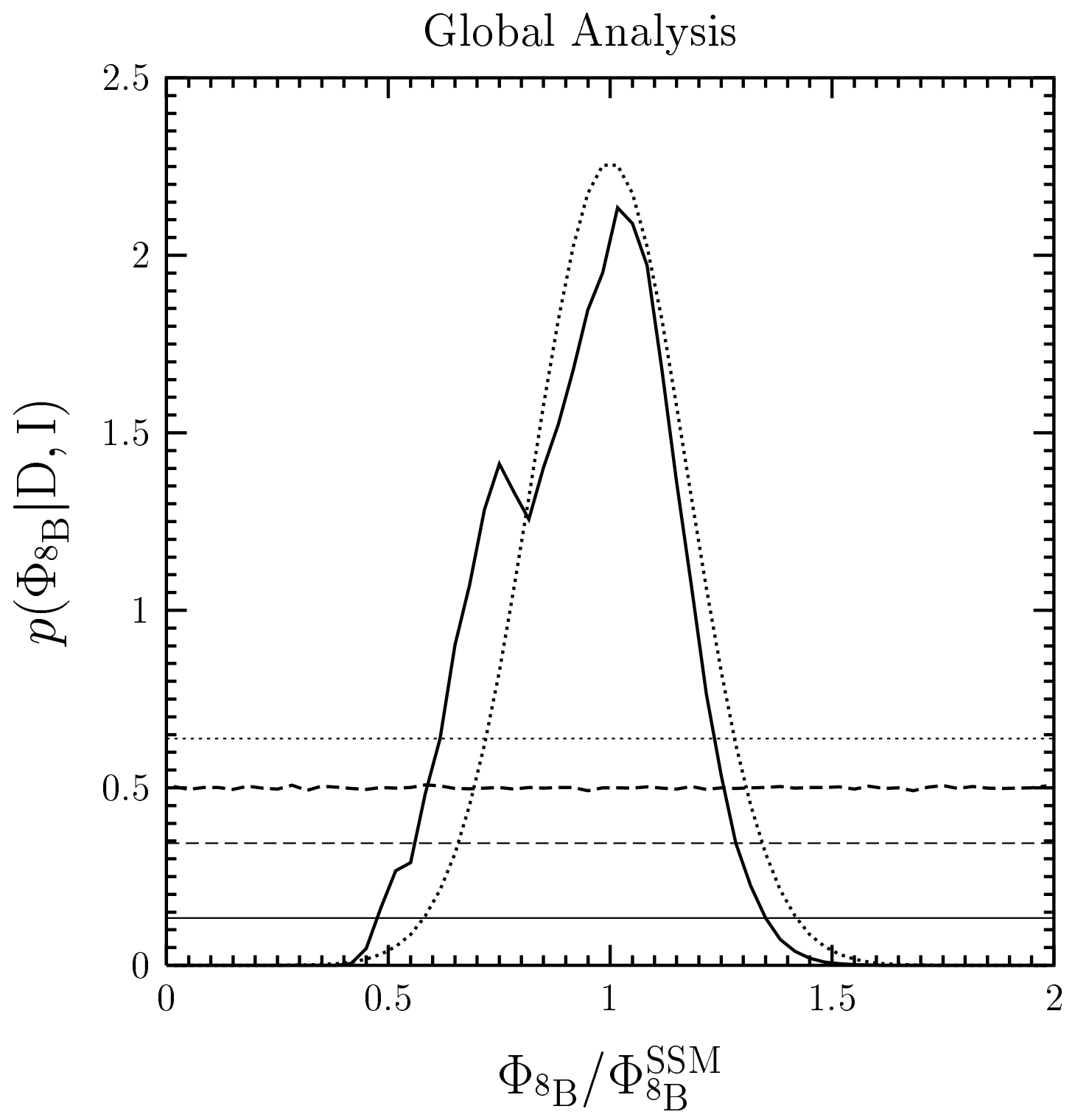

FIG. 20: The thick solid line is the posterior distribution of the ${ }^{8} \mathrm{~B}$ flux obtained with the model independent Global Analysis. The intervals in which the thick solid line lies above the horizontal thin dotted, dashed, and solid lines have, respectively, $90 \%$, $95 \%$ and $99 \%$ posterior probability to contain the true value of the $p p$ flux. The gaussian thick dotted curve is the BP2000 SSM distribution and the thick dashed curve is the prior distribution in the model independent analysis. 